R.

DOCUMENT UI UE 26.327

RESEARCH LA: VATORY OF ELECTRONICS

MASSACHSEI :

E. CAMBRIDGE, MÁSSACHUSETTS 02139, U.S.A.
Loor

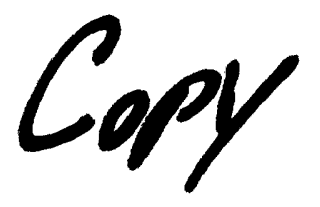

\title{
THE FORCE DENSITY IN POLARIZABLE AND MAGNETIZABLE FLUIDS
}

\author{
L. J. CHU \\ H. A. HAUS \\ P. PENFIELD, JR.
}

$\mathbf{R}$

E

P

O

R

$T$

4

3

3

TECHNICAL REPORT 433

JUNE ।, 1965

MASSACHUSETTS INSTITUTE OF TECHNOLOGY

RESEARCH LABORATORY OF ELECTRONICS

CAMBRIDGE, MASSACHUSETTS 
The Research Laboratory of Electronics is an interdepartmental laboratory in which faculty members and graduate students from numerous academic departments conduct research.

The research reported in this document was made possible in part by support extended the Massachusetts Institute of Technology, Research Laboratory of Electronics, by the JOINT SERVICES ELECTRONICS PROGRAMS (U.S. Army, U.S. Navy, and U.S. Air Force) under Contract No. DA36-039-AMC-03200(E).

Reproduction in whole or in part is permitted for any purpose of the United States Government. 


\title{
MASSACHUSETTS INSTITUTE OF TECHNOLOGY \\ RESEARCH LABORATORY OF ELECTRONICS
}

Technical Report 433

June 1,1965

\section{THE FORCE DENSITY IN POLARIZABLE AND MAGNETIZABLE FLUIDS}

L. J. Chu, H. A. Haus, and P. Penfield, Jr.

(Manuscript received December 23, 1964)

\begin{abstract}
The force density in polarizable and magnetizable fluids in nonuniform motion is evaluated in a way that is consistent with the special theory of relativity. The derivation is based on a generalization of the principle of virtual work. For application of the principle, it is necessary to know the energy density and power-flow density in a local rest frame of the fluid element under consideration. These are obtained from thermodynamic information and an extrapolation of the Poynting theorem applied to a rigid, nondeforming medium. Two different formulations of electrodynamics of moving and deforming media are compared. It is shown that they lead to the same force density if the same thermodynamic information is used in each of them. A simple model for the force density is obtained, starting from the E-H formulation.
\end{abstract}




\section{TABLE OF CONTENTS}

I. Introduction 1

II. Equations of Electromagnetism in the Minkowski Formulation 3

III. Equations of Electromagnetism in the E-H Formulation 5

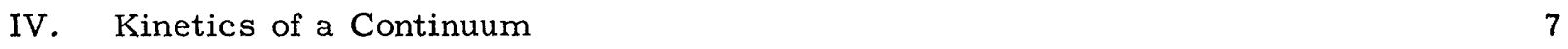

V. Macroscopic Force Density for a Fluid of Charged Particles 9

VI. Relativistic Transformation Laws 12

6. I Transformation of Length and Time 12

6.2 Transformation of Current Density and Charge Density 15

6. 3 Transformation of Fields 16

6. 4 Transformation of Force and Related Quantities 18

$\begin{array}{ll}\text { VII. Principle of Virtual Power } & 20\end{array}$

VIII. Principle of Virtual Power Applied to the Minkowski Formulation 23

IX. Principle of Virtual Power Applied to E-H Formulation 28

X. Interpretation of the Pressure 33

XI. Model of a Polarizable Fluid $\quad 35$

$\begin{array}{ll}\text { Appendix } & 37\end{array}$

$\begin{array}{ll}\text { References } & 39\end{array}$ 


\section{INTRODUCTION}

This report deals with the problem of predicting the force of electromagnetic origin in moving and deforming material bodies. Our theory takes account of the special theory of relativity and treats materials with fairly general electrical and mechanical constitutive relations. Viscosity and intrinsic angular momentum are the most important physical phenomena that are not included. For simplicity we shall discuss, in detail, only polarizable and magnetizable fluids. The same technique can also be applied to solid bodies that are moving and being deformed.

The theoretical bases of electromagnetism and of continuum mechanics are well established. We are interested here in the interaction between electromagnetic fields and mechanical motion; therefore, these well-established theories must be augmented. Several formulations of electromagnetism of moving media have been developed. ${ }^{1-3}$ These formulations appear to be formally different, but all give the same predictions for fields in free space surrounding moving bodies or in slots made (or imagined) in the body for the purpose of measuring fields. The formulations predict different fields inside material bodies, but these different predictions are not in themselves contradictory, since such fields are not directly accessible to measurement. ${ }^{l}$ Indeed, measurements of the deflection of particles injected into the material cannot be used to determine the macroscopic fields inside the material because the average of the microscopic fields seen by the particle is not necessarily the same as the average fields used in the macroscopic analysis; in fact, the former depends upon the velocity of the particle. Thus, any one of these theories formulates the effect of moving matter upon the field correctly if it predicts the correct fields in the space surrounding the moving fluid.

To account for the effects of the polarization and magnetization of moving matter upon its motion, the theory of continuum mechanics is augmented by forces of electromagnetic origin. Since the theory of electromagnetism is not consistent with nonrelativistic mechanics, we shall use relativistic continuum mechanics. For example, see Møller. ${ }^{4}$ The force equation ${ }^{5}$ of relativistic continuum mechanics which we shall use is

$$
\frac{\partial}{\partial t} n m \bar{v}+\nabla \cdot(n m \bar{v} \bar{v})=\bar{f}_{k^{\prime}}
$$

where $\mathrm{nm}$ is the relativistic mass density, $\overline{\mathrm{v}}$ is the velocity, $\bar{f}_{\mathrm{k}}$ is the force per unit volume, and $\partial / \partial t$ is the partial time derivative. The aim of this report is to determine explicit expressions for $\bar{f}_{k}$ under a variety of conditions.

The equations describing moving and deforming continua in the presence of electromagnetic fields consist, on the one hand, of Maxwell's equations and the appropriate electroma'gnetic constitutive laws (which may involve mechanical variables), and, on the other hand, of Newton's law (I) and a specification of the way $\bar{f}_{k}$ is related to mechanical and electromagnetic variables. The equations have not been determined before in the generality that we envision. Many workers have derived what they call a force density, 
or a force of electromagnetic origin (or, the equivalent, an electromagnetic energymomentum tensor), but fail to state explicitly the mechanical equation in which this force, or energy-momentum tensor, is to appear. The various forces or energymomentum tensors did not always agree with each other, and there has been considerable discussion about which of these, if any, is "correct." 6 Such discussions are irrelevant unless the particular force or tensor is accompanied by a statement of the mechanical equation in which it is to be used to predict motion and deformation of the continuum.

Some workers have appreciated this point, and have accounted for it either by explicitly giving Newton's law or by giving the total energy-momentum tensor, the sum of a kinetic tensor and an electromagnetic tensor. Of these workers, only Meixner and his associates $^{7-10}$ have obtained results that agree with ours in the common area of application. Meixner has treated the thermodynamic aspect more thoroughly than we have, but the only rest-frame electrical constitutive relations that he has considered are linear, We allow for nonlinear relations, partly for greater generality, but also for easier physical interpretation of many terms. We also permit constitutive relations that describe dispersive media. We believe our derivation of the force density, as given in this paper, uses fewer and more reasonable postulates than those of Meixner and de $\mathrm{Sa},{ }^{7}$ and also requires somewhat less complicated mathematics. Furthermore, we were able to reconcile the apparently different force densities predicted by the different formulations of electromagnetism of moving media. This is important because some of the theories have rather simple physical interpretations in terms of microscopic models, and these models help toward an understanding of the force density.

In Section II we give the equations of electrodynamics in the Minkowski formulation, and in Section III those of the E-H formulation. In Section IV we study the kinetics of a continuum. In Section V we discuss the force density, energy flow, and momentum flow for a continuum of free charges and currents in the presence of electromagnetic fields. We discuss the generalizations necessary when matter is present. In Section VI we derive the necessary relativistic transformation laws. It is assumed that the reader is familiar with the basic coordinate transformations of special relativity. However, fourvector and tensor notation is not used and four-space geometry is not employed. In Section VII we introduce the principle of virtual power, a generalization of the well-known principle of virtual work. In Section VIII the principle of virtual power is applied to the Minkowski formulation to obtain the force density and power-conversion density for a moving, deforming, nondispersive, polarizable and magnetizable medium. In Section IX the same principle is applied to the $\mathrm{E}-\mathrm{H}$ formulation of electrodynamics, and it is shown that the same force is obtained provided the same thermodynamic information is used. Finally, in Sections X and XI the pressure and force density are interpreted, the latter in terms of a microscopic model. In the Appendix it is shown that the force predicted by the Minkowski and $\mathrm{E}-\mathrm{H}$ formulations are equivalent. 


\section{EQUATIONS OF ELECTROMAGNETISM IN THE MINKOWSKI FORMULATION}

In this section we summarize the equations for electromagnetic fields in the presence of polarizable and magnetizable matter. We present the Minkowski formulation first because it is better known. The E- $\mathrm{H}$ formulation appears in Section III.

Minkowski's equations for the electromagnetic field ${ }^{1}$ are

$$
\begin{aligned}
& \nabla \times \overline{\mathrm{E}}+\frac{\partial \overline{\mathrm{B}}}{\partial \mathrm{t}}=0 \\
& \nabla \cdot \overline{\mathrm{B}}=0 \\
& \nabla \times \overline{\mathrm{H}}-\frac{\partial \overline{\mathrm{D}}}{\partial \mathrm{t}}=\bar{J}_{\mathrm{f}} \\
& \nabla \cdot \overline{\mathrm{D}}=\rho_{\mathrm{f}} .
\end{aligned}
$$

The terms $\bar{J}_{f}$ and $\rho_{f}$ are the free current density and charge density associated with conduction current and convection of the charge. They obey the continuity law

$$
\nabla \cdot \bar{J}_{\mathrm{f}}+\frac{\partial}{\partial \mathrm{t}} \rho_{\mathrm{f}}=0 \text {. }
$$

In the Minkowski formulation the same field equations (2) are used to describe the electromagnetic field, whether in vacuum or in the presence of polarizable and magnetizable matter. The influence of matter is incorporated in the constitutive laws.

The constitutive laws depend, in general, upon the velocity of the matter. Equations (2) and the constitutive laws determine the electromagnetic field, provided the velocity field $\vec{v}(\vec{r}, t)$ of the matter is known. In order to predict the velocity, one needs to know the forces acting upon the medium. The determination of the force density is the main goal of this report. Because certain insights may be gained from the study of fields in free space with free charges, we shall consider this in some detail first. This study serves as a useful introduction to the E-H formulation that is considered in Section III and to the study of the forces on charges in free space considered in Section V.

In free space, the constitutive laws are

$$
\begin{aligned}
& \bar{D}=\epsilon_{0} \overline{\mathrm{E}} \\
& \overline{\mathrm{B}}=\mu_{0} \overline{\mathrm{H}},
\end{aligned}
$$

where $\epsilon_{0}$ is the permittivity of free space, and $\mu_{0}$ is the permeability of free space. When these constitutive relations are used in Maxwell's equations, we find that only two fields, rather than four, are necessary. If we choose $\overline{\mathrm{E}}$ and $\overline{\mathrm{H}}$ as the two fields to use, Maxwell's equations become

$$
\begin{aligned}
& \nabla \times \overline{\mathrm{E}}+\frac{\partial}{\partial \mathrm{t}} \mu_{0} \overline{\mathrm{H}}=0 \\
& \nabla \cdot \mu_{0} \overline{\mathrm{H}}=0
\end{aligned}
$$




$$
\begin{aligned}
& \nabla \times \overline{\mathrm{H}}-\frac{\partial}{\partial t} \epsilon_{0} \overline{\mathrm{E}}=\bar{J}_{\mathrm{f}} \\
& \nabla \cdot \epsilon_{0} \overline{\mathrm{E}}=\rho_{\mathrm{f}^{\prime}}
\end{aligned}
$$

The fields $\overline{\mathrm{E}}$ and $\overline{\mathrm{H}}$ are completely determined by this set of equations, once $\bar{J}_{\mathrm{f}}$ and $\rho_{\mathrm{f}}$ are known as functions of space and time. Equations (5) will be used in Section V to discuss a system of charged particles in free space. They also serve, however, to indicate the philosophy behind the E-H formulation. Instead of retaining the form of the equations of electrodynamics when introducing matter into the vacuum, one may instead treat the changes wrought in the $\mathrm{E}$ and $\mathrm{H}$ fields as being due to the sources (electric or magnetic) brought in by the matter and introduce them explicitly on the right-hand sides of these equations. This approach results in the E-H formulation. The equations that are obtained and the field variables within polarized and magnetized moving matter are different from those of the Minkowski formulation. 


\section{EQUATIONS OF ELECTROMAGNETISM IN THE E-H FORMULATION}

In the E-H formulation only two field vectors, $\overline{\mathrm{E}}$ and $\overline{\mathrm{H}}$, are used in free space. The effect of matter upon the field is taken into account through the polarization density vector $\overline{\mathrm{P}}$ and magnetization density vector $\overline{\mathrm{M}}$ (as observed in the laboratory frame). The equations of electromagnetism include on the right-hand side modifications due to the presence of matter as sources of the field.

$$
\begin{aligned}
& \nabla \times \overline{\mathrm{E}}+\mu_{0} \frac{\partial \overline{\mathrm{H}}}{\partial \mathrm{t}}=-\frac{\partial}{\partial \mathrm{t}} \mu_{0} \overline{\mathrm{M}}-\nabla \times\left(\mu_{0} \overline{\mathrm{M}} \times \overline{\mathrm{v}}\right) \\
& \nabla \cdot \mu_{0} \overline{\mathrm{H}}=-\nabla \cdot \mu_{0} \overline{\mathrm{M}} \\
& \nabla \times \overline{\mathrm{H}}-\epsilon_{0} \frac{\partial \overline{\mathrm{E}}}{\partial \mathrm{t}}=\frac{\partial}{\partial t} \overline{\mathrm{P}}+\nabla \times(\overline{\mathrm{P}} \times \overline{\mathrm{v}})+\overline{J_{f}} \\
& \nabla \cdot \epsilon_{0} \overline{\mathrm{E}}=-\nabla \cdot \overline{\mathrm{P}}+\rho_{\mathrm{f}} .
\end{aligned}
$$

Note that the left-hand sides of (6) and of the equations of the Minkowski formulation in free space (5a) are identical and that the right-hand sides become identical when $\bar{M}$ and $\overline{\mathrm{P}}$ are zero.

It should be pointed out that the $\overline{\mathrm{E}}$ and $\overline{\mathrm{H}}$ vector fields of the present formulation are not the same as those of the Minkowski formulation inside matter if $\bar{M}$ and $\bar{P}$ are nonzero. In fact, comparison of (6) and (2) shows that both sets of equations contain the same information if one sets

$$
\begin{aligned}
& \overline{\mathrm{E}}_{\mathrm{M}}=\overline{\mathrm{E}}+\mu_{0} \overline{\mathrm{M}} \times \overline{\mathrm{v}} \\
& \overline{\mathrm{B}}_{\mathrm{M}}=\mu_{0}(\overline{\mathrm{H}}+\overline{\mathrm{M}}) \\
& \overline{\mathrm{H}}_{\mathrm{M}}=\overline{\mathrm{H}}-\overline{\mathrm{P}} \times \overline{\mathrm{v}} \\
& \overline{\mathrm{D}}_{\mathrm{M}}=\epsilon_{0} \overline{\mathrm{E}}+\overline{\mathrm{P}}
\end{aligned}
$$

provided the free charge density $\rho_{f}$ and free current density $\bar{J}_{f}$ are taken to be the same in the two formulations. Our notation does not distinguish the two sets of field quantities except in those cases where confusion could arise. Then we denote all Minkowski quantities by the subscript $\bar{M}$, and use no subscript for the field quantities of the E-H formulation.

The polarization and magnetization are determined from two constitutive laws relating $\overline{\mathrm{P}}$ and $\overline{\mathrm{M}}$ to $\overline{\mathrm{E}}$ and $\overline{\mathrm{H}}$ and, maybe, their derivatives or integrals, and the geometric state of the matter. 
In free space, the Minkowski $\overline{\mathrm{E}}$ and $\overline{\mathrm{H}}$ fields are identical with those of the $\mathrm{E}-\mathrm{H}$ formulation. Thus both formulations predict the same fields in the space surrounding polarizable and magnetizable matter and in slots cut into the matter, provided equivalent constitutive relations and the same velocity fields are assumed in both. The velocity fields predicted by the two formulations will be the same if the force densities are the same. The force densities will be shown to be the same in the Appendix. 


\section{KINETICS OF A CONTINUUM}

Consider a continuum, such as a fluid or deforming solid, whose motion is described by a velocity field $\bar{v}(\bar{r}, t)$. It is convenient to obtain the equations for such a continuum as the limit of a system of particles of density $n$, with relativistic mass $m$, because then some of the transformation laws become particularly clear. The relativistic equation of motion ( 1 ) is

$$
\nabla \cdot n m \bar{v} \bar{v}+\frac{\partial}{\partial t} n m \bar{v}=\bar{f}_{k}
$$

where the relativistic mass $m$ is related to the rest mass $m_{0}$ by

$$
\mathrm{m}=\frac{\mathrm{m}_{0}}{\sqrt{1-\frac{v^{2}}{c^{2}}}} .
$$

The continuity equation (law of conservation of particles) is

$$
\frac{\partial}{\partial t} n+\nabla \cdot n \bar{v}=0
$$

If the fluid consists of many species of particles that are clustered together so that a single velocity field describes the macroscopic motion, the same approach is applicable. The mass density $\mathrm{nm}$ retains its meaning in this case. The particle density is replaced by quantity $\frac{\mathrm{nm}}{\langle\mathrm{m}\rangle}$ where $\langle\mathrm{m}\rangle$ is the average mass per particle.

One vector equation and one scalar equation describe the kinetics of the system. A different scalar equation, which may be used instead of the continuity equation, is obtained by dot-multiplying (8) by $\bar{v}$ and using (9) and (10):

$$
\nabla \cdot n m c^{2} \bar{v}+\frac{\partial}{\partial t} n m c^{2}=\bar{f}_{k} \cdot \bar{v} .
$$

This is the equation of conservation of energy, $\mathrm{nmc}^{2}$ being the relativistic (kinetic plus rest) energy density of the fluid.

Equations (8) and (11) express conservation of momentum and energy. In (8) $n m \bar{v} \bar{v}=$ $\overline{\mathrm{T}}_{\mathrm{k}}$ is the dyadic density of kinetic momentum flow and $n m \bar{v}=\overline{\mathrm{G}}_{\mathrm{k}}$ is the kinetic momentum density; in (11) $\mathrm{nmc}^{2} \overline{\mathrm{v}}=\overline{\mathrm{S}}_{\mathrm{k}}$ is the density of kinetic energy flow, and $\mathrm{nmc}^{2}=\mathrm{W}_{\mathrm{k}}$ is the kinetic (including rest) energy density. Using these symbols, one may recast (8) and (11) in the form

$$
\begin{aligned}
& \nabla \cdot \overline{\bar{T}}_{k}+\frac{\partial}{\partial t} \bar{G}_{k}=\bar{f}_{k} \\
& \nabla \cdot \overline{\mathrm{S}}_{k}+\frac{\partial}{\partial t} W_{k}=\phi_{k}
\end{aligned}
$$

where

$$
\phi_{\mathrm{k}}=\overline{\mathrm{f}}_{\mathrm{k}} \cdot \overline{\mathrm{v}}
$$


is the power conversion density into kinetic form. Conservation equations of the form (12) may be written for forms of momentum and energy other than kinetic. Because the force density appears on the right-hand side of Eq. (12a), this equation and the remaining similar conservation equations for the electromagnetic field and the internal energy flow in the matter provide the means for determining the force density.

In the next section we arrive at the well-known expression for the force density for a simple physical system, a pressureless charged fluid in an electromagnetic field. The derivation will help us interpret the steps we shall use later to arrive at the force density in a more general medium. 


\section{MACROSCOPIC FORCE DENSITY FOR A FLUID OF CHARGED PARTICLES}

Consider a fluid of charged particles with zero dipole moment moving through free space. The equations of the Minkowski formulation and those of the $\mathrm{E}-\mathrm{H}$ formulation become identical. The force density acting on the particles is obtained directly from the Lorentz Law

$$
\bar{f}_{k}=\rho_{f} \bar{E}+\bar{J}_{f} \times \mu_{0} \bar{H}
$$

where

$$
\overline{J_{f}}=\rho_{f} \bar{v} .
$$

This force density appears on the right-hand side of the momentum equation (8).

The momentum law (8) and the energy law (11) (which adds the same information as the continuity equation) describe the motion of the fluid in terms of the electromagnetic fields. Equations (5) and (15) describe the fields in terms of the motion (velocity field $\bar{v}$ ) of the fluid. Thus, the pressureless fluid of free charge is completely described by these two sets of equations.

It is informative to study the laws of conservation of momentum and energy for the present example. The fluid obeys the momentum law (8) and energy law (11). The electromagnetic field in turn obeys momentum and energy laws. The momentum law is obtained by cross-multiplying (5a) and (5c) by $\epsilon_{0} \overline{\mathrm{E}}$ and $\mu_{0} \overline{\mathrm{H}}$ respectively, and adding. The left-hand side $(\nabla \times \overline{\mathrm{E}}) \times \epsilon_{0} \overline{\mathrm{E}}+(\nabla \times \overline{\mathrm{H}}) \times \mu_{0} \overline{\mathrm{H}}$ can be expressed as a divergence using (5b) and ( $5 \mathrm{~d})$ so that a momentum conservation theorem results:

$$
\begin{aligned}
\nabla \cdot & {\left[\frac{1}{2} \overline{\bar{\delta}}\left(\mu_{0} \overline{\mathrm{H}}^{2}+\epsilon_{0} \overline{\mathrm{E}}^{2}\right)-\left(\epsilon_{0} \overline{\mathrm{E}} \overline{\mathrm{E}}+\mu_{0} \overline{\mathrm{H}} \overline{\mathrm{H}}\right)\right]+\frac{\partial}{\partial \mathrm{t}}\left(\epsilon_{0} \mu_{0} \overline{\mathrm{E}} \times \overline{\mathrm{H}}\right) } \\
& =-\left(\rho_{\mathrm{f}} \overline{\mathrm{E}}+\bar{J}_{\mathrm{f}} \times \mu_{0} \overline{\mathrm{H}}\right) \\
& =-\bar{f}_{\mathrm{k}}
\end{aligned}
$$

Dot multiplication of $(5 a)$ and $(5 c)$ by $-\overline{\mathrm{H}}$ and $\overline{\mathrm{E}}$ respectively and addition yields Poynting's theorem

$$
\begin{aligned}
\nabla \cdot(\overline{\mathrm{E}} \times \overline{\mathrm{H}})+\frac{\partial}{\partial t}\left(\frac{1}{2} \epsilon_{0} \overline{\mathrm{E}}^{2}+\frac{1}{2} \mu_{0} \overline{\mathrm{H}}^{2}\right) & =-\overline{\mathrm{E}} \cdot \overline{\mathrm{J}}_{\mathrm{f}} \\
& =-\overline{\mathrm{f}}_{\mathrm{k}} \cdot \overline{\mathrm{v}}
\end{aligned}
$$

where (14) and (15) were used.

Equations (16) and (17) are the electromagnetic counterparts of the momentum and energy laws of the kinetic system, (8) and (11). One may write them in a form analogous to (12)

$$
\nabla \cdot \overline{\bar{T}}_{e}+\frac{\partial}{\partial t} \bar{G}_{e}=\bar{f}_{e}
$$




$$
\nabla \cdot \bar{S}_{\mathrm{e}}+\frac{\partial}{\partial t} W_{e}=\phi_{e} \cdot
$$

Here

$$
\begin{aligned}
& \overline{\bar{T}}_{e}=\frac{1}{2} \overline{\bar{\delta}}\left(\mu_{0} \overline{\mathrm{H}}^{2}+\epsilon_{0} \overline{\mathrm{E}}^{2}\right)-\left(\epsilon_{0} \overline{\mathrm{E}} \overline{\mathrm{E}}+\mu_{0} \overline{\mathrm{H}} \overline{\mathrm{H}}\right) \\
& \overline{\mathrm{G}}_{\mathrm{e}}=\epsilon_{0} \mu_{0} \overline{\mathrm{E}} \times \overline{\mathrm{H}} \\
& \overline{\mathrm{S}}_{\mathrm{e}}=\overline{\mathrm{E}} \times \overline{\mathrm{H}} \\
& \mathrm{W}_{\mathrm{e}}=\frac{1}{2} \epsilon_{0} \overline{\mathrm{E}}^{2}+\frac{1}{2} \mu_{0} \overline{\mathrm{H}}^{2} \\
& \overline{\mathrm{f}}_{\mathrm{e}}=-\left(\rho_{\mathrm{f}} \overline{\mathrm{E}}+\overline{\mathrm{J}}_{\mathrm{f}} \times \mu_{0} \overline{\mathrm{H}}\right) \\
& \phi_{\mathrm{e}}=-\overline{\mathrm{E}} \cdot \overline{\mathrm{J}}_{\mathrm{f}} .
\end{aligned}
$$

In the present simple case $\overline{\mathrm{f}}_{\mathrm{e}}=-\overrightarrow{\mathrm{f}}_{\mathrm{k}}$ and $\phi_{\mathrm{e}}=-\phi_{\mathrm{k}}$. Thus, adding (12) and (18) one obtains

$$
\begin{aligned}
& \nabla \cdot\left(\overline{\bar{T}}_{\mathrm{e}}+\overline{\bar{T}}_{\mathrm{k}}\right)+\frac{\partial}{\partial \mathrm{t}}\left(\overline{\mathrm{G}}_{\mathrm{e}}+\overline{\mathrm{G}}_{\mathrm{k}}\right)=0 \\
& \nabla \cdot\left(\overline{\mathrm{S}}_{\mathrm{e}}+\overline{\mathrm{S}}_{\mathrm{k}}\right)+\frac{\partial}{\partial t}\left(\mathrm{~W}_{\mathrm{e}}+\mathrm{W}_{\mathrm{k}}\right)=0 .
\end{aligned}
$$

These two conservation relations are in the form of conservation relations with no sources of momentum or energy. Such a conservation relation must be obeyed if a complete description of a system which includes all momenta and energies is used. Equation (18) is typical of conservation theorems obeyed by a partial description not including all momentum and energy terms of the system. The source densities, $\bar{f}_{e}$ and $\phi_{\mathrm{e}}$ of momentum and energy, account for the effects upon the electromagnetic part of the system by the kinetic part.

The derivation of the force densities and conservation principles was simple in the present case with a fluid of noninteracting particles in an electromagnetic field. The force density was obtained from the Lorentz law because the electromagnetic force acts directly toward acceleration of the fluid. In a fluid with pressure or a medium containing polarization and magnetization, the force density cannot be computed directly from a Lorentz-like law: in part, because there are contributions to the force density from particle interactions of classical, as well as quantum-mechanical, nature; and in part because the use of a macroscopic field quantity implies an averaging process. The Lorentz force density is the product of two macroscopic quantities, charge density (or current density) and a macroscopic field. The force itself, even if it were legitimately computed on a nonquantum basis, would be the average of a product of microscopic quantities. An average of a product is not necessarily equal to a product of averages. We conclude that, additional information beyond Maxwell's equations, the Lorentz law, and the relativistic Newton's law is needed to determine the force density or motion and 
deformation in a system in which interaction forces are important because of the high density of the particles. In a nondissipative system such information is macroscopically given once the internal energy density and power-flow density are known. From them one may evaluate the momentum-flow density $\overline{\bar{T}}_{e}$, and the momentum density $\bar{G}_{e}$. These in turn determine the force density through an equation of the form (25a). 


\section{RELATIVISTIC TRANSFORMATION LAWS}

Relativistic transformation laws are needed in a derivation of force density in moving matter for two reasons: (a) The constitutive laws are usually stated in the rest frame, a frame instantaneously at rest with respect to the material element under consideration. The constitutive laws in the laboratory frame of observation are derived from those in the rest frame by relativistic transformations. (b) The derivation of the force density from thermodynamic information leads naturally to the force density as observed in the rest frame. The force density in the laboratory frame is then obtained by a transformation.

\section{1 TRANSFORMATION OF LENGTH AND TIME}

The basic transformation relations, on which all others are based, relate the space and time coordinates in two frames of reference. If the primed reference frame moves with respect to the unprimed one at a velocity $\bar{u}$ then one has 11,12

$$
\begin{aligned}
& \bar{r}^{\prime}=\gamma\left(\bar{r}_{\|}-\bar{u} t\right)+\bar{r}_{\perp} \\
& t^{\prime}=\gamma\left(t-\frac{\bar{u} \cdot \bar{r}}{c^{2}}\right) \\
& \bar{r}=\gamma\left(\bar{r}_{\|}^{\prime}+\bar{u} t^{\prime}\right)+\bar{r}_{\perp}^{\prime} \\
& t=\gamma\left(t^{\prime}+\frac{\bar{u} \cdot \bar{r}^{\prime}}{c^{2}}\right)
\end{aligned}
$$

where

$$
\gamma=\frac{1}{\sqrt{1-\frac{u^{2}}{c^{2}}}} .
$$

Here the subscripts $\|$ and $\perp$ indicate components of vectors parallel and perpendicular to the relative velocity $\bar{u}$.

Equation (26) predicts the Lorentz contraction of a measuring stick at rest in the primed frame (now denoted by the superscript 0 ) when viewed from the unprimed frame, which moves at a velocity $\bar{v}=-\bar{u}$ with respect to the rest frame.

$$
\bar{d}=\sqrt{1-\frac{v^{2}}{c^{2}}} \bar{d}_{\|}^{0}+\bar{d}_{\perp}^{0} .
$$

A more complicated equation results where the measuring rod is compared in two frames, and neither is the rest frame of the rod. This transformation relation is useful for the determination of the transformation of polarization and magnetization density. 


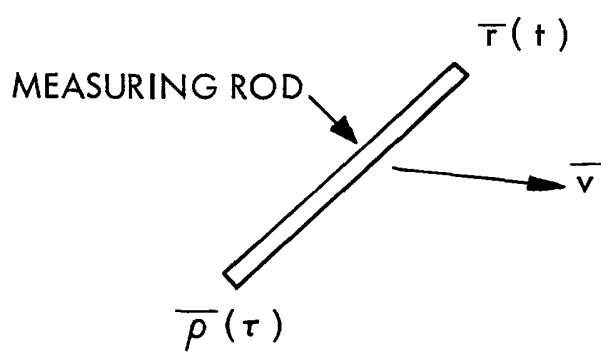

Fig. 1. Measuring rod moving at an arbitrary velocity.

Since it is less well known, we go through the derivation here. The motion of the two endpoints of the measuring rod in the unprimed frame is described by (see Fig. 1)

$$
\begin{aligned}
& \bar{r}=\bar{k}+\bar{v} t \\
& \bar{\rho}=\bar{\kappa}+\bar{v} r,
\end{aligned}
$$

where $\bar{\kappa}$ and $\bar{k}$ are constant vectors. The vector length of the rod as measured in this frame is obtained from the above by setting $t=t$.

$$
\bar{d}=\bar{r}(t)-\bar{\rho}(t)=\bar{k}-\bar{\kappa} .
$$

We next apply (26a) to (29). If the rod is to be measured in the primed frame, the positions of the endpoints of the rod must be recorded simultaneously, i.e., $t^{\prime}=\tau^{\prime}$. The vector difference of the two endpoints is then the vector length $\overline{\mathrm{d}}$.

$$
\begin{aligned}
\bar{d}^{\prime} & =\bar{r}^{\prime}\left(t^{\prime}\right)-\bar{\rho}^{\prime}\left(t^{\prime}\right) \\
& =\bar{r}_{\perp}(t)-\bar{\rho}_{\perp}(\tau)+\gamma\left[\bar{r}_{\|}(t)-\bar{\rho}_{\|}(\tau)\right]-\gamma \bar{u}[t-\tau]
\end{aligned}
$$

where the right-hand side is evaluated at the times $t$ and $T$ corresponding to simultaneity in the primed frame, $t^{\prime}=\tau^{\prime}$. The time difference in the unprimed frame for events simultaneous in the primed frame is obtained from the transformation (26b) applied to $t^{\prime}$ and $\tau^{\prime}$ and setting $t^{\prime}=\tau^{\prime}$.

$$
\begin{aligned}
t-\left.\tau\right|_{t^{\prime}=\tau^{\prime}} & =[\bar{r}(t)-\bar{\rho}(\tau)] \cdot \frac{\bar{u}}{c^{2}} \\
& =\bar{d} \cdot \frac{\bar{u}}{c^{2}}+[\bar{\rho}(t)-\bar{\rho}(\tau)] \cdot \frac{\bar{u}}{c^{2}} \\
& =\bar{d} \cdot \frac{\bar{u}}{c^{2}}+\bar{v} \cdot \frac{\bar{u}}{c^{2}}(t-\tau)
\end{aligned}
$$

and thus 


$$
t-\left.\tau\right|_{t^{\prime}=\tau^{\prime}}=\frac{\bar{d} \cdot \frac{\bar{u}}{c^{2}}}{1-\frac{\bar{u} \cdot \bar{v}}{c^{2}}} .
$$

Introducing this expression into (31) and noting that $\bar{\rho}$ is a linear function of $\tau$ according to (29), one finds

$$
\bar{d}^{\prime}=\frac{\bar{d}_{\perp}+\frac{\bar{d}_{\|}}{\gamma}-\frac{\bar{u} \times(\bar{d} \times \bar{v})}{c^{2}}}{1-\frac{\bar{u} \cdot \bar{v}}{c^{2}}} .
$$

For the special case in which the primed frame is the rest frame, $\bar{v}=\bar{u},(34)$ reduces to (28).

The transformations of space coordinates and time may also be applied to derive the relativistic velocity transformation. A velocity $\bar{v}^{\prime}$ of a point in the primed frame is related to the observed velocity $\bar{v}$ of the point in the unprimed frame by ${ }^{13}$

$$
\overline{v^{\prime}}=\frac{\bar{v}_{11}-\bar{u}+\frac{\bar{v}_{\perp}}{\gamma}}{1-\frac{\bar{u} \cdot \bar{v}}{c^{2}}} .
$$

From (26) and (27) the laws of transformation for the gradient and time derivative follow directly. In order to evaluate the derivative $\nabla_{\|}$parallel to the velocity $\bar{u}$ in the primed frame, one writes $\overline{r_{\|}^{\prime}}$ in terms of $\bar{r}$ and $t$ and uses the chain rule of differentiation. In this way one finds

$$
\begin{aligned}
& \nabla^{\prime}=\gamma\left(\nabla_{I 1}+\frac{\overline{\mathrm{u}}}{\mathrm{c}^{2}} \frac{\partial}{\partial \mathrm{t}}\right)+\nabla_{\perp} \\
& \frac{\partial}{\partial \mathrm{t}^{\prime}}=\gamma\left(\frac{\partial}{\partial \mathrm{t}}+\overline{\mathrm{u}} \cdot \nabla\right) .
\end{aligned}
$$

Next we turn to the transformation relations of the mass and particle density. Since the number of particles observed is an invariant, particle density transforms inversely as a volume element. Consider a set of particles moving with the common velocity $\bar{v}$ in the unprimed reference frame and occupying a volume $V$. It is convenient to use for $V$ a rectangular parallelepiped with one side along $\bar{u}$, the velocity of the primed frame, as seen from the unprimed frame. Application of (34) to the three sides of this parallelepiped yields the transformation law

$$
\mathrm{V}^{\prime}=\frac{\mathrm{V} / \mathrm{\gamma}}{1-\frac{\overline{\mathrm{u}} \cdot \overline{\mathrm{v}}}{\mathrm{c}^{2}}} .
$$


Thus, the transformation law for particle density is

$$
n^{\prime}=\gamma n\left(1-\frac{\bar{u} \cdot \bar{v}}{c^{2}}\right) \text {. }
$$

The mass of a particle with rest mass $m_{0}$ is, in a frame in which the particle moves with the velocity $\bar{v}$,

$$
\mathrm{m}=\frac{\mathrm{m}_{0}}{\sqrt{1-\frac{\mathrm{v}^{2}}{\mathrm{c}^{2}}}} .
$$

Using the transformation law for velocity, (35), one may find the mass $\mathrm{m}^{\prime}$ as observed in the primed frame

$$
m^{\prime}=\gamma m\left(1-\frac{\bar{u} \cdot \bar{v}}{c^{2}}\right) \text {. }
$$

\subsection{TRANSFORMATION OF CURRENT DENSITY AND CHARGE DENSITY}

The transformation law of electric current density and charge density follows directly from the laws of transformation for particle density and velocity. Let us consider a current density $\overline{\mathrm{J}}$ produced by a single species of charges of charge density $\rho$ flowing at a velocity $\bar{v}$. Then

$$
\bar{J}=\rho \bar{v} .
$$

Since $\rho=q n$, where $q$ is the charge of one particle and a relativistic invariant and $n$ is the particle density, we may use transformations (35) and (38) to obtain

$$
\begin{aligned}
& \overline{J^{\prime}}=\gamma\left(\bar{J}_{\|}-\bar{u} \rho\right)+\bar{J}_{\perp} \\
& \rho^{\prime}=\gamma\left(\rho-\frac{\bar{u} \cdot \bar{J}}{c^{2}}\right) .
\end{aligned}
$$

The derivation of the transformation laws was based on a model involving one simple species of charges. In this special case it is possible to find a reference frame in which the current density vanishes (the rest frame of the charge particles), but it is not possible to find a frame in which the charge density vanishes. In four-space terminology this means that $\bar{J}$ and $\rho$ form a timelike four-vector. The transformation law is, as yet, limited to a special case. However, it is easily generalized to apply to a general current flow (for which one might find reference frames in which the charge density vanishes) by applying the transformation to current densities and charge densities that are the superpositions of the flows of different species. Applying (42) to each species and adding, one finds laws still of the form of (42), but not limited to a single species. 


\section{3 TRANSFORMATION OF FIELDS}

In the Minkowski formalism the transformation laws for the fields are ${ }^{14}$

$$
\begin{aligned}
& \overline{\mathrm{E}}^{\prime}=\overline{\mathrm{E}}_{\|}+\gamma\left(\overline{\mathrm{E}}_{\perp}+\overline{\mathrm{u}} \times \overline{\mathrm{B}}\right) \\
& \overline{\mathrm{H}}^{\prime}=\overline{\mathrm{H}}_{\|}+\gamma\left(\overline{\mathrm{H}}_{\perp}-\overline{\mathrm{u}} \times \overline{\mathrm{D}}\right) \\
& \overline{\mathrm{D}}^{\prime}=\overline{\mathrm{D}}_{\|}+\gamma\left(\overline{\mathrm{D}}_{\perp}+\frac{\overline{\mathrm{u}} \times \overline{\mathrm{H}}}{\mathrm{c}^{2}}\right) \\
& \overline{\mathrm{B}}^{\prime}=\overline{\mathrm{B}}_{\|}+\gamma\left(\overline{\mathrm{B}}_{\perp}-\frac{\overline{\mathrm{u}} \times \overline{\mathrm{E}}}{c^{2}}\right) .
\end{aligned}
$$

When one applies the transformation laws to constitutive laws stated in the rest frame of the material, one identifies the primed frame with the rest frame and $\bar{u}$ with the velocity $\bar{v}$ of the rest frame with respect to the laboratory frame. In this special case we shall identify the quantity $\gamma$ by a subscript 0 .

$$
\gamma_{0}=\frac{1}{\sqrt{1-\frac{v^{2}}{c^{2}}}} .
$$

In the frame instantaneously at rest with respect to the volume element of the medium under study (the rest frame), one class of constitutive laws is

$$
\begin{aligned}
& \overline{\mathrm{D}}^{0}=\overline{\mathrm{D}}^{0}\left(\overline{\mathrm{E}}^{0}\right) \\
& \overline{\mathrm{B}}^{0}=\overline{\mathrm{B}}^{0}\left(\overline{\mathrm{H}}^{0}\right) .
\end{aligned}
$$

Here $\overline{\mathrm{D}}^{0}\left(\overline{\mathrm{E}}^{0}\right)$ and $\overline{\mathrm{B}}^{0}\left(\overline{\mathrm{H}}^{0}\right)$ are in general nonlinear vector functions of $\overline{\mathrm{E}}^{0}$ and $\overline{\mathrm{H}}^{0}$. These equations imply that the polarization and magnetization depend upon the $\overline{\mathrm{E}}^{0}$ and $\overline{\mathrm{H}}^{0}$ fields respectively at the instant of interest and at the point of interest. These laws do not describe materials in which the polarization may be affected by the $\overline{\mathrm{H}}^{0}$ field and the magnetization by the $\overline{\mathrm{E}}^{0}$ field and further, do not include space-dispersive and timedispersive media in which the polarization or magnetization depend on space or time derivatives of the electromagnetic fields.

In order to use (45) in Maxwell's equations in the laboratory frame, with respect to which the medium moves, one makes use of the transformations (43) relating the field variables in the rest frame (identified with the primed frame) to those in the unprimed frame when the former moves at a velocity $\overline{\mathrm{V}}$ with respect to the latter.

The transformation laws in the E-H formulation ${ }^{15}$ are similar to a certain extent to those of the Minkowski formulation. In particular, since the left-hand sides of Maxwell's equations for the $\mathrm{E}-\mathrm{H}$ formulation are identical with those of the Minkowski formulation in free space, it is clear that the E-H fields of the former (even in the presence of 
polarized matter) should transform like the latter in free space, with $\overline{\mathrm{B}}=\mu_{0} \overline{\mathrm{H}}, \overline{\mathrm{D}}=\epsilon_{0} \overline{\mathrm{E}}$. One has,

$$
\begin{aligned}
& \overline{\mathrm{E}^{\prime}}=\overline{\mathrm{E}}_{\|}+\gamma\left(\overline{\mathrm{E}}_{\perp}+\overline{\mathrm{u}} \times \mu_{0} \overline{\mathrm{H}}\right) \\
& \overline{\mathrm{H}}^{\prime}=\overline{\mathrm{H}}_{\|}+\gamma\left(\overline{\mathrm{H}}_{\perp}-\overline{\mathrm{u}} \times \epsilon_{0} \overline{\mathrm{E}}\right) .
\end{aligned}
$$

There are no quantities analogous to $\overline{\mathrm{D}}$ and $\overline{\mathrm{B}}$ in the $\mathrm{E}-\mathrm{H}$ formulation. Instead there are $\overline{\mathrm{P}}$ and $\overline{\mathrm{M}}$. The transformation for $\overline{\mathrm{P}}$ may be constructed by applying physical reasoning to a model for polarization. The polarization density in any frame may be written as the product

$$
\overline{\mathrm{P}}=\mathrm{Nq} \overline{\mathrm{d}}
$$

where $\overline{\mathrm{d}}$ is the vector distance separating the charges $\pm \mathrm{q}$, and $\mathrm{N}$ is the dipole number density.

The transformation law for vector distance (34), the law for particle density (38), and the fact that charge is an invariant under relativistic transformations yield immediately the transformation for polarization density

$$
\begin{aligned}
\overline{\mathrm{P}}^{\prime} & =N^{\prime} \mathrm{q} \overline{\mathrm{d}}^{\prime} \\
& =\gamma N q\left[\overline{\mathrm{d}}_{\perp}+\frac{\overline{\mathrm{d}}_{\|}}{\gamma}-\frac{\overline{\mathrm{u}} \times(\overline{\mathrm{d}} \times \overline{\mathrm{v}})}{\mathrm{c}^{2}}\right]
\end{aligned}
$$

or

$$
\overline{\mathrm{P}}^{\prime}=\overline{\mathrm{P}}_{\|}+\gamma \overline{\mathrm{P}}_{\perp}-\gamma \frac{\overline{\mathrm{u}} \times(\overline{\mathrm{P}} \times \overline{\mathrm{v}})}{\mathrm{c}^{2}} .
$$

Since the magnetization density appears in Maxwell's equation of the E-H formulation in a similar way, one expects that it should transform in a way similar to that of $\bar{P}$. One finds indeed

$$
\bar{M}^{\prime}=\bar{M}_{\|}+\gamma \bar{M}_{\perp}-\gamma \frac{\bar{u} \times(\bar{M} \times \bar{v})}{c^{2}} .
$$

It is of interest to compare the Minkowski transformations and those of the $\mathrm{E}-\mathrm{H}$ formulation. Equation (7) expresses the Minkowski variables $\bar{E}_{M}, \bar{H}_{M}, \bar{D}_{M}$ and $\bar{B}_{M}$ in terms of the $\overline{\mathrm{E}}$ and $\overline{\mathrm{H}}$ field of the $\mathrm{E}-\mathrm{H}$ formulation, and the polarization $\overline{\mathrm{P}}$ and $\overline{\mathrm{M}}$. One may check easily that the transformation laws for the Minkowski variables are consistent with those of the E-H formulation by transforming the left-hand sides of (7) by the Minkowski transformations (43) and the right-hand sides by the transformations (46), (49), and (50) of the E-H formulation.

The next question to be resolved is whether the same fields are predicted by both formulations either in the free space surrounding moving matter or in slots cut into 
moving matter. In order to evaluate the fields in either formulation, it is necessary to use constitutive laws relating $\overline{\mathrm{D}}_{M}$ and $\overline{\mathrm{B}}_{M}$ to $\overline{\mathrm{E}}_{M}$ and $\overline{\mathrm{H}}_{M}$ in the Minkowski formulation and relating $\overline{\mathrm{P}}$ and $\overline{\mathrm{M}}$ to $\overline{\mathrm{E}}$ and $\overline{\mathrm{H}}$ in the $\mathrm{E}-\mathrm{H}$ formulation. An example of such constitutive laws is given in (45) for the Minkowski formulation for the case of nondispersive polarizable and magnetizable matter. These laws are applied in the rest frame and then transformed into the moving frame by the relativistic transformation (43).

Corresponding constitutive laws may be applied in the $\mathrm{E}-\mathrm{H}$ formulation. In the rest frame the quantities in the two formulations are simply related

$$
\begin{aligned}
& \overline{\mathrm{D}}_{\mathrm{M}}^{0}=\epsilon_{0} \overline{\mathrm{E}}^{0}+\overline{\mathrm{P}}^{0} \\
& \overline{\mathrm{E}}_{\mathrm{M}}^{0}=\overline{\mathrm{E}}^{0} \\
& \overline{\mathrm{B}}_{\mathrm{M}}^{0}=\mu_{0}\left(\overline{\mathrm{H}}^{0}+\overline{\mathrm{M}}^{0}\right) \\
& \overline{\mathrm{H}}_{\mathrm{M}}^{0}=\overline{\mathrm{H}}^{0} .
\end{aligned}
$$

Thus, if one sets

$$
\begin{aligned}
& \overline{\mathrm{P}}^{0}\left(\overline{\mathrm{E}}^{0}\right)=\overline{\mathrm{D}}_{\mathrm{M}}^{0}\left(\overline{\mathrm{E}}^{0}\right)-\epsilon_{0} \overline{\mathrm{E}}^{0} \\
& \mu_{0} \overline{\mathrm{M}}^{0}\left(\overline{\mathrm{H}}^{0}\right)=\overline{\mathrm{B}}_{\mathrm{M}}^{0}\left(\overline{\mathrm{H}}^{0}\right)-\mu_{0} \overline{\mathrm{H}}^{0}
\end{aligned}
$$

where $\bar{D}_{M}^{0}\left(\overline{\mathrm{E}}^{0}\right)$ and $\overline{\mathrm{B}}_{\mathrm{M}}^{0}\left(\overline{\mathrm{H}}^{0}\right)$ are the specific functional dependencies of (45), the constitutive laws as well as the four Maxwell's equations of the $\mathrm{E}-\mathrm{H}$ formulation can be cast into the Minkowski form by using (7) since the velocity field $\bar{v}$ has the same meaning in both formulations. Since $\overline{\mathrm{E}}_{\mathrm{M}}=\overline{\mathrm{E}}$ and $\overline{\mathrm{H}}_{\mathrm{M}}=\overline{\mathrm{H}}$ in free space the two formulations predict the same fields in free space surrounding the medium. The predictions of velocity are the same if the force densities of the two formulations are identical. We shall demonstrate this in the Appendix.

The arguments presented here are applicable to other than nondispersive materials provided the correspondence between the constitutive laws of the two formulations is maintained in the manner illustrated above.

\section{4 TRANSFORMATION OF FORCE AND RELATED QUANTITIES}

The transformation of force density and power-conversion density may be obtained by recognizing the transformation of the quantity $\overline{\mathrm{f}}=\rho \overline{\mathrm{E}}_{M}+\overline{\mathrm{J}} \times \overline{\mathrm{B}}_{M}$, which is a force density in the Minkowski formulation, and $\phi=\overline{\mathrm{E}}_{\mathrm{M}} \cdot \overline{\mathrm{J}}$, which is a power-conversion density. By using the transformation laws (42) and (43) one finds

$$
\bar{f}^{\prime}=\gamma\left(\bar{f}_{\|}-\frac{\bar{u} \phi}{c^{2}}\right)+\bar{f}_{\perp}
$$




$$
\phi^{\prime}=\gamma(\phi-\bar{u} \cdot \bar{f}) .
$$

Note that the vector $\bar{f}$ and scalar $\phi / c^{2}$ transform like the space coordinate $\bar{r}$ and time $t$.

Next we consider the transformation of momentum density $\overline{\mathrm{G}}$ and momentum-flow density $\overline{\overline{\mathrm{T}}}$, of energy density $\mathrm{W}$ and energy-flow density $\overline{\mathrm{S}}$. These quantities appear in conservation relations of the form (12) or (18) which we repeat here in a general form

$$
\begin{aligned}
& \nabla \cdot \overline{\overline{\mathrm{T}}}+\frac{\partial}{\partial t} \overline{\mathrm{G}}=\overline{\mathrm{f}} \\
& \nabla \cdot \overline{\mathrm{S}}+\frac{\partial}{\partial \mathrm{t}} \mathrm{W}=\phi .
\end{aligned}
$$

A force density and power-conversion density appear on the right-hand side of these equations. The transformation relations for these are (53) and the transformations of the operators $\nabla$ and $\partial / \partial t$ are (36). If one requires that the conservation relations (54) remain in the same form in any inertial frame, one finds the transformation laws for $\overline{\bar{T}}, \overline{\mathrm{G}}, \overline{\mathrm{S}}$, and $\mathrm{W}$ are

$$
\begin{gathered}
\overline{\bar{T}}^{\prime}=\overline{\bar{T}}_{\perp \perp}+\gamma\left(\overline{\bar{T}}_{\| \perp}-\bar{u}_{\perp}\right)+\gamma\left(\overline{\bar{T}}_{\perp \|}-\frac{\bar{S}_{\perp} \bar{u}}{c^{2}}\right)+\gamma^{2}\left(\overline{\bar{T}}_{\|\|}-\bar{u} \bar{G}_{\|}-\frac{\bar{S}_{\|} \bar{u}}{c^{2}}+\frac{\bar{u} \bar{u} W}{c^{2}}\right) \\
\bar{G}^{\prime}=\gamma\left(\bar{G}_{\perp}-\frac{\bar{u} \cdot \overline{\bar{T}}_{\| \perp}}{c^{2}}\right)+\gamma^{2}\left(\bar{G}_{\|}-\frac{\bar{u} \cdot \overline{\bar{T}}_{\|\|}}{c^{2}}-\frac{\bar{u} W}{c^{2}}+\frac{\bar{u}(\bar{u} \cdot \bar{S})}{c^{4}}\right) \\
\bar{S}^{\prime}=\gamma\left(\bar{S}_{\perp}-\overline{\bar{T}}_{\perp \|} \cdot \bar{u}\right)+\gamma^{2}\left[\bar{S}_{\|}-\overline{\bar{T}}_{\|\|} \cdot \bar{u}-\bar{u} W+\bar{u}(\bar{u} \cdot \bar{G})\right] \\
W^{\prime}=\gamma^{2}\left(W-\bar{u} \cdot \bar{G}-\frac{\bar{u} \cdot \bar{S}}{c^{2}}+\frac{\bar{u} \cdot \overline{\bar{T}} \cdot \bar{u}}{c^{2}}\right)
\end{gathered}
$$

where the subscripts \|\|$,\|\perp, \perp\|$, and $\perp \perp$ refer to components of a tensor with the first or second index parallel or perpendicular to the transformation velocity $\bar{u}$. These equations may be put to test by applying them to the kinetic quantities $\overline{\mathrm{T}}_{\mathrm{k}}, \overline{\mathrm{G}}_{\mathrm{k}}, \overline{\mathrm{S}}_{\mathrm{k}}$, and $\mathrm{W}_{\mathrm{k}}$ and to the electromagnetic quantities $\overline{\bar{T}}_{\mathrm{e}}, \overline{\mathrm{G}}_{\mathrm{e}}, \overline{\mathrm{S}}_{\mathrm{e}}, \mathrm{w}_{\mathrm{e}}$. As derived here, the laws are of greater generality, because they apply to cases where $\bar{S} \neq \bar{G} / c^{2}$, whereas both the kinetic and free-space electromagnetic example satisfy this relation.

We have now provided the necessary background for a statement of the principle of virtual power, and its application to the derivation of force density in moving matter. 


\section{PRINCIPLE OF VIRTUAL POWER}

In the application of the principle of virtual work, one assumes that the medium is subject to a (virtual) deformation by an external agent (this agent may be a set of Maxwell demons properly placed within the medium). The change in the energy is the work performed by the agent causing the deformation. This work is, in turn, directly related to the stresses within the medium against which the deformation is performed.

In this section we shall develop a more general formalism for the application of the principle of virtual work. In particular, allowance will be made for power supplied during the deformation, a generalization not usually treated in the literature. It is convenient to treat the deformation on a time-rate basis, and thus we use the name principle of virtual power. We shall make use of the energy-conservation equation which, when applied to a virtual rate of deformation of the medium under consideration, will enable us to evaluate the momentum density and stress (or momentum-flow) tensor from knowledge of the energy density, power-flow density, and power-conversion density in the rest frame of the medium.

It is often convenient to separate the description of a deforming medium into separate characterizations of its kinetic properties, its electromagnetic properties, its thermodynamic properties, etc. Each of these partial descriptions obeys a set of conservation equations of the form

$$
\begin{aligned}
& \nabla \cdot \overline{\bar{T}}+\frac{\partial \overline{\mathrm{G}}}{\partial \mathrm{t}}=\overline{\mathrm{f}} \\
& \nabla \cdot \overline{\mathrm{S}}+\frac{\partial \mathrm{W}}{\partial \mathrm{t}}=\phi
\end{aligned}
$$

where $\overline{\bar{T}}$ is the stress tensor, $\bar{G}$ the momentum density, $\bar{f}$ the force density, $\bar{S}$ the powerflow density, $W$ the energy density, and $\phi$ the power-conversion density. The quantities $\bar{f}$ and $\phi$ connect this partial description to the remaining partial descriptions. The principle of virtual power may be applied to any of these partial descriptions. Examples of partial conservation equations like (56) are given earlier for the kinetic aspects (12) and for electromagnetic fields in free space (18).

In applying the principle of virtual power to a partial description, we assume the form of $\overline{\mathrm{S}}, \mathrm{W}$, and $\phi$ in the rest frame is known, i.e., that we know the form of $\overline{\mathrm{S}}^{0}, \mathrm{~W}^{0}$, and $\phi^{0}$. We also assume knowledge of the differential equations obeyed by the system. Then the transformation laws (55) are sufficient to enable us to calculate $\overline{\bar{T}}, \bar{G}, \bar{S}, W, \bar{f}$ and $\phi$ in arbitrary frames.

We wish to investigate (56b). Evaluation of the derivatives $\nabla \cdot \bar{S}$ and $\partial W / \partial t$ in (56b) requires knowledge of $\bar{S}(\bar{x}, t)$ and $W(\bar{x}, t)$ whereas we know only $\bar{S}^{0}(\bar{x}, t)$ and $W^{0}(\bar{x}, t)$. The relation between them, to first order in $\bar{v}$, is

$$
W(\bar{x}, t)=W^{0}(\bar{x}, t)+\bar{v}(\bar{x}, t) \cdot \bar{G}^{0}(\bar{x}, t)+\frac{\bar{v}(\bar{x}, t) \cdot S^{0}(\bar{x}, t)}{c^{2}}
$$




$$
\bar{S}(\bar{x}, t)=\bar{S}^{0}(\bar{x}, t)+\overline{\bar{T}}^{0}(\bar{x}, t) \cdot \bar{v}(\bar{x}, t)+W^{0}(\bar{x}, t) \bar{v}(\bar{x}, t) .
$$

Note that (57b) has a simple nonrelativistic interpretation. The "power-flow" density $\overline{\mathrm{S}}$ in a frame with respect to which the medium moves has to include, in addition to the rest-frame power-flow density $\overline{\mathrm{S}}^{0}$, the convection of energy $\overline{\mathrm{vW}}^{0}$, and the rate at which work is performed by the stresses represented by $\overline{\bar{T}}^{0}$.

Consider a material element at the point $\bar{x}^{0}$ at time $t^{0}$. The rest frame corresponding to that point at that time is the inertial frame for which $\bar{v}\left(\bar{x}^{0}, t^{0}\right)=0$. For convenience we shall call this the "virtual" frame. Note, however, that in the "virtual" frame, $\nabla \bar{v}$ and $\partial \bar{v} / \partial t$ need not vanish. We assume that virtual deformations can be applied to the material to produce arbitrary $\nabla \overline{\mathrm{v}}$ and $\partial \overline{\mathrm{v}} / \partial \mathrm{t}$ in the "virtual" frame at this point, but keep $\overline{\mathrm{v}}\left(\overline{\mathrm{x}}^{0}, \mathrm{t}^{0}\right)=0$.

Introducing (57) into (56b), collecting the terms involving $\phi^{0}, \mathrm{~W}^{0}$, and $\overline{\mathrm{S}}^{0}$ on one side, and evaluating for the point $\overline{\mathrm{x}}^{0}$ and time $\mathrm{t}^{0}$ in the "virtual" frame (so that $\partial \overline{\mathrm{v}} / \partial \mathrm{t}$ and $\nabla \overline{\mathrm{v}}$ need not be zero), one obtains

$$
\nabla \cdot \overline{\mathrm{S}}^{0}+\frac{\overline{\mathrm{S}}^{0}}{\mathrm{c}^{2}} \cdot \frac{\partial \overline{\mathrm{v}}}{\partial \mathrm{t}}+\frac{\partial}{\partial \mathrm{t}} \mathrm{W}^{0}+\mathrm{W}^{0} \nabla \cdot \overline{\mathrm{v}}-\phi^{0}=-\overline{\overline{\mathrm{T}}}^{0} \cdot \nabla \overline{\mathrm{v}}-\overline{\mathrm{G}}^{0} \cdot \frac{\partial \overline{\mathrm{v}}}{\partial \mathrm{t}} .
$$

where the colon denotes the double dot product between two tensors; in subscript notation, $\overline{\bar{A}}: \overline{\bar{B}}=A_{i j} B_{i j}$. An alternative form for (58) can be found from the law of conservation of particles (10), applied in the virtual frame

$$
\frac{\partial n^{0}}{\partial t}+n^{0} \nabla \cdot \bar{v}=0
$$

Thus, substitution of (59) into (58) yields

$$
\nabla \cdot \overline{\mathrm{S}}^{0}+\mathrm{n}^{0} \frac{\partial}{\partial \mathrm{t}}\left(\frac{\mathrm{W}^{0}}{\mathrm{n}^{0}}\right)+\frac{\overline{\mathrm{S}}^{0}}{\mathrm{c}^{2}} \cdot \frac{\partial \overline{\mathrm{v}}}{\partial \mathrm{t}}-\phi^{0}=-\overline{\overline{\mathrm{T}}}^{0}: \nabla \overline{\mathrm{v}}-\overline{\mathrm{G}}^{0} \cdot \frac{\partial \overline{\mathrm{V}}}{\partial \mathrm{t}} .
$$

We suppose now that we know $\overline{\mathrm{S}}^{0}, \partial\left(\mathrm{W}^{0} / \mathrm{n}^{0}\right) / \partial t$, and $\phi^{0}$ in terms of the pertinent physical variables (for example, particle density, polarization density), at least to first order in the derivatives of $\bar{v}$. We may introduce the appropriate expressions for these quantities into the left-hand side of (58) or $(60)$. Usually $\overline{\mathrm{S}}^{0}, \partial\left(\mathrm{W}^{0} / \mathrm{n}^{0}\right) / \partial \mathrm{t}$ and $\phi^{0}$ are found in terms of the physical variables in the actual rest frame and not the "virtual" frame, which is not a rest frame at all points and all times. In order to apply the derivatives in (58) or (60), it is first necessary to express all physical variables appearing in $\bar{S}^{0}, \partial\left(W^{0} / n^{0}\right) / \partial t$ and $\phi^{0}$ in terms of their values observed in the "virtual" frame, by first-order transformation laws of the sort discussed in Section VI.

When this is done, some terms on the left-hand side of (58) or (60) contain $\nabla \bar{v}$ or $\partial \bar{v} / \partial t$ and some do not. One may then apply the equations of motion in the "virtual" frame to eliminate those terms that do not contain $\nabla \bar{v}$ and $\partial \bar{v} / \partial t$, a process that will, in general, introduce new terms that contain $\nabla \bar{v}$ and $\partial \bar{v} / \partial t$. (This is why one cannot simply omit the zero-order terms.) By consistently neglecting second-order terms in 
$\nabla \bar{v}$ or $\partial \bar{v} / \partial t$, we have thus reduced the left-hand side of $(58)$ or $(60)$ to the form

$$
\nabla \cdot \overline{\mathrm{S}}^{0}+\frac{\overline{\mathrm{S}}^{0}}{\mathrm{c}^{2}} \cdot \frac{\partial \overline{\mathrm{v}}}{\partial \mathrm{t}}+\mathrm{n}^{0} \frac{\partial}{\partial \mathrm{t}}\left(\frac{\mathrm{W}^{0}}{\mathrm{n}^{0}}\right)-\phi^{0}=\bar{\alpha} \cdot \frac{\partial \overline{\mathrm{v}}}{\partial \mathrm{t}}+\overline{\bar{\beta}}: \nabla \overline{\mathrm{v}}
$$

for some $\bar{\alpha}$ and $\overline{\bar{\beta}}$.

Because the space and time derivatives of $\bar{v}$ can, by hypothesis, be adjusted arbitrarily and independently in the "virtual" frame, (61) and (60) can be identified term by term so that $\overline{\bar{T}}^{0}$ and $\overline{\mathrm{G}}^{0}$ are related to the derived $\bar{a}$ and $\overline{\bar{\beta}}$ as

$$
\begin{aligned}
& \overline{\mathrm{G}}^{0}=-\overline{\bar{a}} \\
& \overline{\overline{\mathrm{T}}}^{0}=-\overline{\bar{\beta}} .
\end{aligned}
$$

In this way one obtains the stress (or momentum-flow) tensor and the momentum density to zero order in $\bar{v}$ and the derivatives of $\bar{v}$. It should be noted that this method does not yield contributions to the stress and momentum that depend upon velocity gradients or accelerations. Viscous forces are of this type. To find such stresses or forces, one needs additional physical information or insight, beyond the knowledge of $\bar{S}^{0}, \partial\left(W^{0} / n^{0}\right) / \partial t$, and $\phi^{0}$ to first orcier in the derivatives of $\bar{v}$.

Suppose, however, that $\overline{\bar{T}}^{0}$ or $\overline{\mathrm{G}}^{0}$ do not contain terms dependent on velocity gradient or acceleration. Then, the stress (momentum-flow) tensor $\overline{\bar{T}}$ and momentum density $\bar{G}$ can be obtained in an arbitrary (laboratory) frame in which the material moves with the velocity $\overline{\mathrm{V}}$ by a relativistic transformation of $\overline{\mathrm{T}}^{0}, \overline{\mathrm{G}}^{0}, \overline{\mathrm{S}}^{0}$, and $\mathrm{W}^{0}$ according to (55). The force density is obtained from $\overline{\bar{T}}$ and $\bar{G}$ by means of (56).

It is worthwhile to summarize the procedure leading to the evaluation of the force density in a series of specific steps. Equation (58) or (60) serve as the starting point. In order to bring the left-hand side into the desired form, one uses the dependences of $\underline{S}^{0}, \partial\left(W^{0} / n^{0}\right) / \partial t$ and $\phi^{0}$ on the physical variables in the rest frame. The resulting equation contains, in general, space and time derivatives of rest-frame variables. To carry out these derivatives conveniently, one needs instead "virtual"-frame variables, which are found in terms of rest-frame variables by means of the transformation laws (to first order in $\overline{\mathrm{v}}$ ) of the variables. In general, some terms now contain the derivatives of $\overline{\mathrm{v}}$ and some do not. Those that do not can be eliminated through the use of the known equations of motion. The result, when evaluated to first order in $\nabla \bar{v}$ and $\partial \bar{v} / \partial t$, holds for arbitrary $\nabla \overline{\mathrm{V}}$ and $\partial \overline{\mathrm{v}} / \partial \mathrm{t}$, and hence (if $\overline{\bar{T}}^{0}$ and $\overline{\mathrm{G}}^{0}$ do not contain $\nabla \overline{\mathrm{V}}$ or $\partial \overline{\mathrm{V}} / \partial \mathrm{t}$ ) we can evaluate $\overline{\bar{T}}^{0}, \bar{G}^{0}$ through term-by-term identification. $\overline{\bar{T}}, \bar{G}, \bar{S}$, and $W$ in the laboratory frame are obtained through the use of the relativistic transformation laws, and the coupling terms $\overline{\mathrm{f}}$ and $\phi$ can be found by application of the conservation laws to find $\overline{\mathrm{f}}$ and $\phi$. 


\section{PRINCIPLE OF VIRTUAL POWER APPLIED TO THE MINKOWSKI FORMULATION}

In the Minkowski formulation one may conveniently subdivide the description of a moving and deforming medium into two parts. One part describes the kinetic properties of the system with conservation principles (12)

$$
\begin{aligned}
& \nabla \cdot \overline{\bar{T}}_{k}+\frac{\partial}{\partial t} \bar{G}_{k}=\bar{f}_{k}, \\
& \nabla \cdot \bar{s}_{k}+\frac{\partial}{\partial t} W_{k}=\phi_{k} .
\end{aligned}
$$

The other part describes the electromagnetic and thermodynamic properties of the system. This description in turn obeys momentum and energy-conservation principles of the form [compare with the free-space expressions (18)]

$$
\begin{aligned}
& \nabla \cdot \overline{\bar{T}}_{M}+\frac{\partial}{\partial t} \bar{G}_{M}=\bar{f}_{M} \\
& \nabla \cdot \bar{S}_{M}+\frac{\partial}{\partial t} W_{M}=\phi_{M} .
\end{aligned}
$$

If the description of the system is complete, it must obey conservation principles for the total momentum and the total energy and no source terms should appear on the right-hand sides of these equations. In other words,

$$
\begin{aligned}
& \nabla \cdot\left(\overline{\bar{T}}_{k}+\overline{\bar{T}}_{M}\right)+\frac{\partial}{\partial t}\left({\overline{G_{k}}}_{k}+\overline{\mathrm{G}}_{M}\right)=0 \\
& \nabla \cdot\left(\overline{\mathrm{S}}_{\mathrm{k}}+\overline{\mathrm{S}}_{\mathrm{M}}\right)+\frac{\partial}{\partial \mathrm{t}}\left(\mathrm{W}_{\mathrm{k}}+\mathrm{W}_{\mathrm{M}}\right)=0,
\end{aligned}
$$

so that

$$
\begin{aligned}
& \bar{f}_{M}=-\bar{f}_{k} \\
& \phi_{M}=-\phi_{k} .
\end{aligned}
$$

It follows from (67) that the kinetic force density $\overline{\mathrm{f}}_{\mathrm{k}}$ may be found, once $\overline{\overline{\mathrm{T}}}_{\mathrm{M}}$ and $\overline{\mathrm{G}}_{\mathrm{M}}$ are known. These are found through the use of the principle of virtual power.

For the application of the principle of virtual power, one needs to know the expression for the time rate of change of the rest-frame energy density, $\partial \mathrm{W}_{\mathrm{M}}^{0} / \partial \mathrm{t}$, the power-flow density in the rest frame, $\overrightarrow{\mathrm{S}}_{\mathrm{M}}^{0}$, and the rest-frame power-conversion density $\phi_{\mathrm{M}}^{0}$. This "thermodynamic" information in turn must be consistent with Poynting's theorem for stationary media. This fact serves as a guide for the form that the energy density and power flow may assume. Poynting's theorem in the rest frame of a stationary lossless material assumes the form

$$
\nabla \cdot(\overline{\mathrm{E}} \times \overline{\mathrm{H}})+\overline{\mathrm{E}} \cdot \frac{\partial \overline{\mathrm{D}}}{\partial \mathrm{t}}+\overline{\mathrm{H}} \cdot \frac{\partial \overline{\mathrm{B}}}{\partial \mathrm{t}}=0 .
$$


We assume that the material is conservative. From Poynting's theorem one then concludes that, since $\overline{\mathrm{E}} \times \overline{\mathrm{H}}$ is to be interpreted as a power-flow density, $\overline{\mathrm{E}} \cdot \mathrm{d} \overline{\mathrm{D}}+\overline{\mathrm{H}} \cdot \mathrm{d} \overline{\mathrm{B}}$ is to be interpreted as a change of stored energy, $\mathrm{dW}_{M}$, in the rest frame of a stationary material. Even for conservative media this interpretation is not necessary. A somewhat more general case is treated in Section IX.

If the material is being deformed, the energy density must also depend on the restframe particle density $n^{0}$; if the process is adiabatic, the change of energy will be

$$
d W_{M}^{0}=\bar{E}^{0} \cdot d \bar{D}^{0}+\bar{H}^{0} \cdot d \bar{B}^{0}+\frac{\partial W^{0}}{\partial n^{0}} d n^{0} .
$$

This is the expression for the change of energy density which is generally accepted. ${ }^{16}$ From (69) one obtains the time-rate of change of the energy directly

$$
\frac{\partial W_{M}^{0}}{\partial t}=\bar{E}^{0} \cdot \frac{\partial \bar{D}^{0}}{\partial t}+\bar{H}^{0} \cdot \frac{\partial \overline{\mathrm{B}}^{0}}{\partial t}+\frac{\partial W_{M}^{0}}{\partial n^{0}} \frac{\partial n^{0}}{\partial t} .
$$

Poynting's theorem (68) further suggests that the power-conversion density $\phi_{M}^{0}$ in the rest frame is zero.

$$
\phi_{\mathrm{M}}^{0}=0 .
$$

The power-flow density in the rest frame has already been identified as

$$
\overline{\mathrm{S}}_{\mathrm{M}}^{0}=\overline{\mathrm{E}}^{0} \times \overline{\mathrm{H}}^{0} \text {. }
$$

Note that the power flow is assumed to be electromagnetic in nature. Heat flow, if present, would have to be added, but we shall limit our investigation to adiabatic processes. We shall accept (70), (71), and (72) as available thermodynamic information. From these, we shall derive the expression for the force density in moving and deforming media. Since no further assumptions will be made, except that the deformation is slow enough (reversible) so that higher order terms in $\bar{v}$ may be disregarded in the rest frame, the predicted force density tests the correctness of these equations.

Making use of the continuity law (59) we obtain for the left-hand side of (58), to first order in $\bar{v}$,

$$
\begin{aligned}
\nabla \cdot & \bar{S}_{M}^{0}+\frac{\bar{S}_{M}^{0}}{c^{2}} \cdot \frac{\partial \bar{v}}{\partial t}+\frac{\partial W_{M}^{0}}{\partial t}+W_{M}^{0} \nabla \cdot \bar{v}-\phi_{M}^{0} \\
= & \nabla \cdot\left(\bar{E}^{0} \times \bar{H}^{0}\right)+\frac{\bar{E}^{0} \times \bar{H}^{0}}{c^{2}} \cdot \frac{\partial \bar{v}}{\partial t} \\
& +\bar{E}^{0} \cdot \frac{\partial \bar{D}^{0}}{\partial t}+. \bar{H}^{0} \cdot \frac{\partial \bar{B}^{0}}{\partial t}+\left(\frac{\partial W_{M}^{0}}{\partial n^{0}}-\frac{W_{M}^{0}}{n^{0}}\right) \frac{\partial n^{0}}{\partial t} \\
& \equiv Q .
\end{aligned}
$$


For convenience, we use the symbol $Q$ to define the expression (73). Next we shall use the transformation relations for the field variables and the appropriate field equations in order to express $Q$ as a quantity involving the virtual velocity field $\bar{v}$ to the first order. The transformation relations for the fields, to first order in $\bar{v}$, are

$$
\begin{aligned}
& \overline{\mathrm{E}}^{0}=\overline{\mathrm{E}}+\overline{\mathrm{v}} \times \overline{\mathrm{B}} \\
& \overline{\mathrm{H}}^{0}=\overline{\mathrm{H}}-\overline{\mathrm{v}} \times \overline{\mathrm{D}} \\
& \overline{\mathrm{D}}^{0}=\overline{\mathrm{D}}+\frac{\overline{\mathrm{v}} \times \overline{\mathrm{H}}}{\mathrm{c}^{2}} \\
& \overline{\mathrm{B}}^{0}=\overline{\mathrm{B}}-\frac{\overline{\mathrm{v}} \times \overline{\mathrm{E}}}{\mathrm{c}^{2}} .
\end{aligned}
$$

It is convenient to introduce the Legendre transform of $W_{M}^{0}$ with respect to $n^{0}, \bar{D}^{0}$ and $\overline{\mathrm{B}}^{0}$,

$$
\mathrm{n}^{0} \frac{\partial \mathrm{W}_{\mathrm{M}}^{0}}{\partial \mathrm{n}^{0}}-\mathrm{W}_{\mathrm{M}}^{0}+\overline{\mathrm{E}}^{0} \cdot \overline{\mathrm{D}}^{0}+\overline{\mathrm{H}}^{0} \cdot \overline{\mathrm{B}}^{0} .
$$

We subtract $(1 / 2) \overline{\mathrm{E}}^{0} \cdot \mathrm{D}^{0}+(1 / 2) \overline{\mathrm{H}}^{0} \cdot \overline{\mathrm{B}}^{0}$ from this transform so as to render it zero in vacuum and use the symbol $\pi_{M}$ to denote the resulting quantity.

$$
\pi_{M}=n^{0} \frac{\partial W_{M}^{0}}{\partial n^{0}}-W_{M}^{0}+\frac{1}{2} \bar{E}^{0} \cdot \bar{D}^{0}+\frac{1}{2} \bar{H}^{0} \cdot \bar{B}^{0} .
$$

Introducing (74) into (73), one finds to first order in $\bar{v}$

$$
\begin{aligned}
\mathrm{Q}= & \nabla \cdot(\overline{\mathrm{E}} \times \overline{\mathrm{H}})+\overline{\mathrm{E}} \cdot \frac{\partial \overline{\mathrm{D}}}{\partial \mathrm{t}}+\overline{\mathrm{H}} \cdot \frac{\partial \overline{\mathrm{B}}}{\partial \mathrm{t}}+\left[\overline{\mathrm{B}} \overline{\mathrm{H}}+\overline{\mathrm{D}} \overline{\mathrm{E}}-\overline{\bar{\delta}}\left(\pi_{\mathrm{M}}+\frac{1}{2} \overline{\mathrm{E}} \cdot \overline{\mathrm{D}}+\frac{1}{2} \overline{\mathrm{H}} \cdot \overline{\mathrm{B}}\right)\right]: \nabla \overline{\mathrm{V}} \\
& -\left[\frac{\partial}{\partial \mathrm{t}}\left(\frac{\overline{\mathrm{v}}}{\mathrm{c}^{2}}\right)\right] \cdot(\overline{\mathrm{E}} \times \overline{\mathrm{H}}) .
\end{aligned}
$$

The first three terms add up to zero because of Maxwell's Eqs. (2). The remaining terms are of first order in $\bar{v}$, and since we evaluate these in the "virtual" frame only at the point $\bar{x}_{0}$ and at time $t_{0}$, we can write the rest-frame values instead. Term-by-term identification of (77) with the right-hand side of (58) yields

$$
\overline{\bar{T}}_{M}^{0}=\overline{\bar{\delta}}\left(\pi_{M}+\frac{1}{2} \overline{\mathrm{E}}^{0} \cdot \overline{\mathrm{D}}^{0}+\frac{1}{2} \overline{\mathrm{H}}^{0} \cdot \overline{\mathrm{B}}^{0}\right)-\overline{\mathrm{B}}^{0} \overline{\mathrm{H}}^{0}-\overline{\mathrm{D}}^{0} \overline{\mathrm{E}}^{0}
$$

and

$$
\bar{G}_{M}^{0}=\frac{\bar{E}^{0} \times \bar{H}^{0}}{c^{2}} .
$$

The resulting expression for a linear nondispersive material is in agreement with 
Eq. 56. 17 of Landau and Lifshitz. ${ }^{17}$

The Lorentz transformation laws of Section VI applied to $\overline{\bar{T}}_{M}^{0}, \bar{G}_{M}^{0}, \bar{S}_{M}^{0}, W_{M}^{0}$ imply

$$
\begin{aligned}
& \overline{\bar{T}}_{M}=\frac{1}{2} \overline{\bar{\delta}}(\bar{B} \cdot \bar{H}+\bar{D} \cdot \bar{E})-\bar{D} \bar{E}-\bar{B} \bar{H}+\pi_{M} \overline{\bar{\delta}}+\gamma_{0}^{2} \frac{\bar{v} \bar{v}}{c^{2}}\left(\pi_{M}+U^{0}\right) \\
& +\gamma_{0}^{2} \bar{v}\left(\frac{\bar{E} \times \bar{H}}{c^{2}}-\bar{D} \times \bar{B}\right)+\gamma_{0}^{2} \frac{\bar{v}}{c} \frac{\bar{v}}{c} \times(\bar{D} \times \bar{E}+\bar{B} \times \bar{H}) \\
& \overline{\mathrm{G}}_{\mathrm{M}}=\gamma_{0}^{2} \frac{\overline{\mathrm{E}} \times \overline{\mathrm{H}}}{\mathrm{c}^{2}}-\gamma_{0}^{2} \frac{\overline{\mathrm{v}}^{2}}{\mathrm{c}^{2}} \overline{\mathrm{D}} \times \overline{\mathrm{B}}+\gamma_{0}^{2} \frac{\overline{\mathrm{v}}}{\mathrm{c}^{2}}\left(\pi_{M}+U^{0}\right) \\
& +\gamma_{0}^{2} \frac{\overline{\mathrm{v}}}{c^{2}} \times(\overline{\mathrm{D}} \times \overline{\mathrm{E}}+\overline{\mathrm{B}} \times \overline{\mathrm{H}}) \\
& \bar{S}_{M}=\bar{E} \times \bar{H}+\gamma_{0}^{2} \bar{v}\left(\pi_{M}+U^{0}\right)+\gamma_{0}^{2} \frac{\bar{v}}{c} \frac{\bar{v}}{c} \cdot\left(\bar{E} \times \bar{H}-c^{2} \bar{D} \times \bar{B}\right) \\
& W_{M}=\frac{1}{2}(\overline{\mathrm{E}} \cdot \overline{\mathrm{D}}+\overline{\mathrm{H}} \cdot \overline{\mathrm{B}})+\gamma_{0}^{2}\left[\mathrm{U}^{0}+\frac{\mathrm{v}^{2}}{\mathrm{c}^{2}} \pi_{M}+\frac{\overline{\mathrm{v}}}{\mathrm{c}^{2}} \cdot\left(\overline{\mathrm{E}} \times \overline{\mathrm{H}}-\mathrm{c}^{2} \overline{\mathrm{D}} \times \overline{\mathrm{B}}\right)\right]
\end{aligned}
$$

where we have defined the quantity

$$
U^{0} \equiv W_{M}^{0}-\frac{1}{2}\left(\bar{E}^{0} \cdot \bar{D}^{0}+\bar{H}^{0} \cdot \bar{B}^{0}\right) .
$$

The force density in the laboratory frame $\bar{f}_{k}$ is obtained from (67a) by using the expressions above for $\overline{\bar{T}}_{M}$ and $\overline{\mathrm{G}}_{\mathrm{M}}$

$$
\begin{aligned}
\bar{f}_{k}= & -\nabla \pi_{M}-\frac{\partial}{\partial t}\left(\bar{D} \times \bar{B}-\bar{R}+\frac{\pi_{M}+U^{0}}{c^{2}-v^{2}} \bar{v}\right) \\
& -\nabla\left(\frac{1}{2} \bar{E} \cdot \bar{D}+\frac{1}{2} \bar{H} \cdot \bar{B}\right) \\
& +\nabla \cdot\left[\bar{D} \bar{E}+\bar{B} \bar{H}+\bar{v} \bar{R}-\frac{\pi_{M}+U^{0}}{c^{2}-v^{2}} \bar{v} \bar{v}\right] .
\end{aligned}
$$

Here, we have defined the quantity

$$
\overline{\mathrm{R}} \equiv \frac{\mathrm{c}^{2} \overline{\mathrm{D}} \times \overline{\mathrm{B}}-\overline{\mathrm{E}} \times \overline{\mathrm{H}}}{\mathrm{c}^{2}-\mathrm{v}^{2}}-\frac{\overline{\mathrm{v}} \times(\overline{\mathrm{B}} \times \overline{\mathrm{H}}+\overline{\mathrm{D}} \times \overline{\mathrm{E}})}{\mathrm{c}^{2}-\mathrm{v}^{2}} .
$$

The power-conversion density $\phi_{\mathrm{k}}$ is

$$
\phi_{\mathrm{k}}=\overline{\mathrm{f}}_{\mathrm{k}} \cdot \overline{\mathrm{v}} \text {. }
$$

The above force density is relatively complicated. A physical interpretation of each of 
the terms is not straightforward. We shall not attempt an interpretation at this point. We shall, instead, interpret the corresponding expression in the $\mathrm{E}-\mathrm{H}$ formulation, which lends itself better to an interpretation on the basis of a simple model of polarization and magnetization. 


\section{PRINCIPLE OF VIRTUAL POWER APPLIED TO E-H FORMULATION}

In the $\mathrm{E}-\mathrm{H}$ formulation (6) the presence of matter is described in terms of sources of the electric and magnetic kind acting on the electromagnetic field just the way free charges and free currents act on the electromagnetic field in free space, provided that one allows for the concepts dual to electric charge and current, magnetic charge and current. Therefore, in the E-H formulation, it is natural to subdivide the description of a moving and deforming medium into three parts. The first part describes the kinetic properties of the system and obeys the conservation laws of kinetic momentum and energy (12)

$$
\begin{aligned}
& \nabla \cdot \overline{\mathrm{T}}_{\mathrm{k}}+\frac{\partial}{\partial \mathrm{t}} \overline{\mathrm{G}}_{\mathrm{k}}=\overline{\mathrm{f}}_{\mathrm{k}} \\
& \nabla \cdot \overline{\mathrm{S}}_{\mathrm{k}}+\frac{\partial}{\partial \mathrm{t}} \mathrm{W}_{\mathrm{k}}=\phi_{\mathrm{k}} .
\end{aligned}
$$

The quantities $\bar{f}_{k}$ and $\phi_{k}$ are as yet unknown. The second part describes the energy and momentum associated with the electric and magnetic fields. Conservation laws of energy and momentum for a fluid of charged particles have been derived (18). A similar derivation in the case of a polarizable and magnetizable matter yields the same equation

$$
\begin{aligned}
& \nabla \cdot \overline{\bar{T}}_{e}+\frac{\partial}{\partial t} \bar{G}_{e}=\bar{f}_{e} \\
& \nabla \cdot \bar{S}_{e}+\frac{\partial}{\partial t} w_{e}=\phi_{e},
\end{aligned}
$$

with $\overline{\mathrm{T}}_{\mathrm{e}}, \overline{\mathrm{S}}_{\mathrm{e}}, \overline{\mathrm{G}}_{\mathrm{e}}$, and $\mathrm{W}_{\mathrm{e}}$ still given by (19)-(22), but now $\overline{\mathrm{f}}_{\mathrm{e}}$ and $\phi_{\mathrm{e}}$ are changed. Now

$$
\begin{aligned}
\overline{\mathrm{f}}_{\mathrm{e}}= & \overline{\mathrm{E}} \nabla \cdot \overline{\mathrm{P}}-\left\{\frac{\partial \overline{\mathrm{P}}}{\partial \mathrm{t}}+\nabla \times(\overline{\mathrm{P}} \times \overline{\mathrm{v}})\right\} \times \mu_{0} \overline{\mathrm{H}}+\overline{\mathrm{H}} \nabla \cdot \mu_{0} \overline{\mathrm{M}} \\
& +\left\{\frac{\partial}{\partial \mathrm{t}} \mu_{0} \overline{\mathrm{M}}+\nabla \times\left(\mu_{0} \overline{\mathrm{M}} \times \overline{\mathrm{v}}\right)\right\} \times \epsilon_{0} \overline{\mathrm{E}}
\end{aligned}
$$

and

$$
\phi_{e}=-\overline{\mathrm{E}} \cdot\left[\frac{\partial \overline{\mathrm{P}}}{\partial \mathrm{t}}+\nabla \times(\overline{\mathrm{P}} \times \overline{\mathrm{v}})\right]-\overline{\mathrm{H}} \cdot\left[\frac{\partial}{\partial \mathrm{t}} \mu_{0} \overline{\mathrm{M}}+\nabla \times\left(\mu_{0} \overline{\mathrm{M}} \times \overline{\mathrm{v}}\right)\right] .
$$

The description of the interaction of matter and field is contained in a set of conservation relations of momentum and energy of the general form

$$
\begin{aligned}
& \nabla \cdot \overline{\bar{T}}_{m}+\frac{\partial}{\partial t} \bar{G}_{m}=\bar{f}_{m} \\
& \nabla \cdot \bar{S}_{m}+\frac{\partial}{\partial t} W_{m}=\phi_{m} .
\end{aligned}
$$

If the above three partial descriptions include all aspects of the medium and of the 
electromagnetic field, then the total momentum flow and momentum density must obey the conservation theorem

$$
\nabla \cdot\left(\overline{\bar{T}}_{k}+\overline{\bar{T}}_{e}+\overline{\bar{T}}_{m}\right)+\frac{\partial}{\partial t}\left(\bar{G}_{k}+\bar{G}_{e}+\bar{G}_{m}\right)=0
$$

which implies that

$$
\overline{\mathrm{f}}_{\mathrm{k}}=-\nabla \cdot\left(\overline{\overline{\mathrm{T}}}_{\mathrm{e}}+\overline{\overline{\mathrm{T}}}_{\mathrm{m}}\right)-\frac{\partial}{\partial \mathrm{t}}\left(\overline{\mathrm{G}}_{\mathrm{e}}+\overline{\mathrm{G}}_{\mathrm{m}}\right) .
$$

In a corresponding way the energy conservation relation

$$
\nabla \cdot\left(\overline{\mathrm{S}}_{\mathrm{k}}+\overline{\mathrm{S}}_{\mathrm{m}}+\overline{\mathrm{S}}_{\mathrm{e}}\right)+\frac{\partial}{\partial \mathrm{t}}\left(\mathrm{W}_{\mathrm{k}}+\mathrm{W}_{\mathrm{m}}+\mathrm{W}_{\mathrm{e}}\right)=0
$$

implies that

$$
\phi_{\mathrm{k}}=-\left(\phi_{\mathrm{e}}+\phi_{\mathrm{m}}\right) .
$$

Thus our problem is solved if we can find $\overline{\bar{T}}_{m}, \bar{G}_{m}, \bar{S}_{m}$, and $W_{m}$. We now find these by means of the principle of virtual power.

To apply the principle of virtual power, we need, for $(60)$, expressions for $\bar{S}_{\mathrm{m}}^{0}$, $\partial\left(\mathrm{W}_{\mathrm{m}}^{0} / \mathrm{n}^{0}\right) / \partial \mathrm{t}$, and $\phi_{\mathrm{m}}^{0}$. First, from $(92)$, since $\phi_{\mathrm{k}}=\overline{\mathrm{f}}_{\mathrm{k}} \cdot \overline{\mathrm{v}}$ we have in the rest frame $\phi_{\mathrm{k}}^{0}=0$, so

$$
\phi_{\mathrm{m}}^{0}=-\phi_{\mathrm{e}}^{0}=\overline{\mathrm{E}}^{0} \cdot\left[\frac{\partial \overline{\mathrm{P}}^{0}}{\partial \mathrm{t}}+\overline{\mathrm{P}}^{0} \nabla \cdot \overline{\mathrm{v}}-\overline{\mathrm{P}}^{0} \cdot \nabla \overline{\mathrm{v}}\right]+\overline{\mathrm{H}}^{0} \cdot\left[\frac{\partial \mu_{0} \overline{\mathrm{M}}^{0}}{\partial \mathrm{t}}+\mu_{0} \overline{\mathrm{M}}^{0} \nabla \cdot \overline{\mathrm{v}}-\mu_{0} \overline{\mathrm{M}}^{0} \cdot \nabla \overline{\mathrm{v}}\right]
$$

which becomes, from (59),

$$
\phi_{m}^{0}=n^{0} \overline{\mathrm{E}}^{0} \cdot \frac{\partial}{\partial t}\left(\frac{\overline{\mathrm{P}}^{0}}{\mathrm{n}^{0}}\right)-\overline{\mathrm{P}}^{0} \overline{\mathrm{E}}^{0}: \nabla \overline{\mathrm{v}}+\mathrm{n}^{0} \overline{\mathrm{H}}^{0} \cdot \frac{\partial}{\partial t}\left(\frac{\mu_{0} \overline{\mathrm{M}}^{0}}{\mathrm{n}^{0}}\right)-\mu_{0} \overline{\mathrm{M}}^{0} \overline{\mathrm{H}}^{0}: \nabla \overline{\mathrm{v}}
$$

Next, consideration of a rigid, stationary, nondeforming medium leads to reasonable expressions for $\overline{\mathrm{S}}_{\mathrm{m}}^{0}$ and $\partial\left(\mathrm{W}_{\mathrm{m}}^{0} / \mathrm{n}^{0}\right) / \partial t$. Thus from (87b)

$$
\phi_{e}=-\bar{E} \cdot \frac{\partial \bar{P}}{\partial t}-\bar{H} \cdot \frac{\partial \mu_{0} \bar{M}}{\partial t} .
$$

But, (88) and (92) imply, since $\phi_{\mathrm{k}}=0$,

$$
\nabla \cdot \bar{S}_{m}+\frac{\partial W_{m}}{\partial t}=\bar{E} \cdot \frac{\partial \bar{P}}{\partial t}+\bar{H} \cdot \frac{\partial \mu_{0} \bar{M}}{\partial t}
$$

For many media operating adiabatically, the right-hand side is a perfect time derivative, and hence we identify 


$$
\frac{\partial W_{m}}{\partial t}=\bar{E} \cdot \frac{\partial \bar{P}}{\partial t}+\bar{H} \cdot \frac{\partial \mu_{0} \bar{M}}{\partial t}
$$

and

$$
\overline{\mathrm{S}}_{\mathrm{m}}=0 \text {. }
$$

Equation (97) is not altered in this case by writing it in the form

$$
n \frac{\partial\left(W_{m} / n\right)}{\partial t}=n \bar{E} \cdot \frac{\partial(\bar{P} / n)}{\partial t}+n \bar{H} \cdot \frac{\partial\left(\mu_{0} \bar{M} / n\right)}{\partial t}
$$

since for stationary media $\mathrm{n}$ is constant.

Conservative media in general are of this type, including dispersive and nondispersive media, and gyroelectric and gyromagnetic media.

These results about stationary media do not prove anything about deforming media, but they suggest reasonable assumptions. First, we retain (98), even though $\nabla \bar{v}$ and $\partial \bar{v} / \partial t$ may not be zero:

$$
\bar{S}_{\mathrm{m}}^{0}=0
$$

And second, to allow for an additional possible dependence of $\mathrm{w}_{\mathrm{m}}^{0}$ upon mechanical variables (for the fluid, only $\mathrm{n}^{0}$ ), we generalize (99) to the form

$$
n^{0} \frac{\partial}{\partial t}\left(\frac{W_{m}^{0}}{n^{0}}\right)=n^{0} \bar{E}^{0} \cdot \frac{\partial}{\partial t}\left(\frac{\bar{P}^{0}}{n^{0}}\right)+n^{0} \bar{H}^{0} \cdot \frac{\partial}{\partial t}\left(\frac{\mu_{0} \bar{M}^{0}}{n^{0}}\right)+\frac{\pi m}{n^{0}} \frac{\partial n^{0}}{\partial t}
$$

where the new variable $\pi_{m}$ will be called the "total pressure."

A word may be in order about (101). As it stands, it holds for media that may be time-dispersive (but not necessarily space-dispersive), gyromagnetic, or gyroelectric, just as long as there is a rest-frame energy density $\mathrm{w}_{\mathrm{m}}^{0}$. It is not assumed that $\mathrm{w}_{\mathrm{m}}^{0}$ is a function of $\overline{\mathrm{P}}^{0}, \overline{\mathrm{M}}^{0}$, and $\mathrm{n}^{0}$, but only that the time derivative of $\mathrm{W}_{m}^{0}$ in the virtual frame be of the form of (101). In dispersive media, for example, $\mathrm{w}_{\mathrm{m}}^{0}$ is not a function of these variables yet its time derivative is of the form stated. We have not made any assumptions as to the form of the constitutive relations between $\overline{\mathrm{E}}^{0}, \overline{\mathrm{H}}^{0}, \pi_{m}, \overline{\mathrm{P}}^{0}, \overline{\mathrm{M}}^{0}$, and $n^{0}$; these relations may in fact involve time derivatives of these variables. Some examples are discussed in Section $\mathbf{X}$.

We are now in a position to apply the principle of virtual power. Thus, $(60)$ becomes

$$
\begin{aligned}
-\overline{\bar{T}}_{m}^{0}: \nabla \bar{v}-\overline{\mathrm{G}}_{\mathrm{m}}^{0} \cdot \frac{\partial \overline{\mathrm{v}}}{\partial \mathrm{t}} & =\nabla \cdot \overline{\mathrm{S}}_{\mathrm{m}}^{0}+\mathrm{n}^{0} \frac{\partial}{\partial \mathrm{t}}\left(\frac{\mathrm{W}_{\mathrm{m}}^{0}}{\mathrm{n}^{0}}\right)+\frac{\overline{\mathrm{S}}_{\mathrm{m}}^{0}}{\mathrm{c}^{2}} \cdot \frac{\partial \overline{\mathrm{v}}}{\partial \mathrm{t}}-\phi_{\mathrm{m}}^{0} \\
& =\frac{{ }^{\pi} \mathrm{m}}{\mathrm{n}} \frac{\partial \mathrm{n}^{0}}{\partial \mathrm{t}}+\overline{\mathrm{P}}^{0} \overline{\mathrm{E}}^{0}: \nabla \overline{\mathrm{v}}+\mu_{0} \overline{\mathrm{M}}^{0} \overline{\mathrm{H}}^{0}: \nabla \overline{\mathrm{v}} .
\end{aligned}
$$

By using the continuity law in the rest frame (59) we put (102) into the form 


$$
-\overline{\bar{T}}_{\mathrm{m}}^{0}: \nabla \overline{\mathrm{v}}-\overline{\mathrm{G}}_{\mathrm{m}}^{0} \cdot \frac{\partial \mathrm{v}}{\partial \mathrm{t}}=\left[\overline{\mathrm{P}}^{0} \overline{\mathrm{E}}^{0}+\mu_{0} \overline{\mathrm{M}}^{0} \overline{\mathrm{H}}^{0}\right]: \nabla \overline{\mathrm{v}}-\pi_{\mathrm{m}} \nabla \cdot \overline{\mathrm{v}}
$$

and term-by-term evaluation shows that

$$
\overline{\overline{\mathrm{T}}}_{\mathrm{m}}^{0}=-\overline{\mathrm{P}}^{0} \overline{\mathrm{E}}^{0}-\mu_{0} \overline{\mathrm{M}}^{0} \overline{\mathrm{H}}^{0}+\pi_{\mathrm{m}} \overline{\bar{\delta}}
$$

and

$$
\overline{\mathrm{G}}_{\mathrm{m}}^{0}=0
$$

Next, we determine the stress tensor $\overline{\bar{T}}_{m}$, the momentum density $\bar{G}_{m}$, the power flow density $\bar{S}_{m}$, and the energy density $W_{m}$ in the laboratory frame by means of the transformation laws (55) with $\bar{u}=-\bar{v}$,

$$
\begin{aligned}
& \overline{\bar{T}}_{m}=\pi_{m} \overline{\bar{\delta}}-\bar{P}\left(\bar{E}+\bar{v} \times \mu_{0} \bar{H}\right)-\mu_{0} \bar{M}\left(\bar{H}-\bar{v} \times \epsilon_{0} \overline{\mathrm{E}}\right)+\bar{v} \bar{G}_{m} \\
& \bar{G}_{m}=\gamma_{0}^{2} \frac{\bar{v}}{c^{2}}\left(\pi m+W_{m}^{0}\right)-\frac{\gamma_{0}^{2}}{c^{2}}\left[(\bar{v} \cdot \bar{P})\left(\bar{E}+\bar{v} x_{\mu_{0}} \bar{H}\right)+\left(\bar{v} \cdot \mu_{0} \bar{M}\right)\left(\bar{H}-\bar{v} \times \epsilon_{0} \bar{E}\right)\right] \\
& \bar{S}_{m}=\gamma_{0}^{2} \bar{v}\left(\pi_{m}+W_{m}^{0}\right)-(\bar{E} \cdot \bar{v}) \bar{P}-(\bar{H} \cdot \bar{v}) \mu_{0} \bar{M}-\gamma_{0}^{2} \frac{\bar{v}}{c^{2}}\left[(\bar{P} \cdot \bar{v})(\bar{E} \cdot \bar{v})+\left(\mu_{0} \bar{M} \cdot \bar{v}\right)(\bar{H} \cdot \bar{v})\right] \\
& W_{m}=\gamma_{0}^{2} W_{m}^{0}+\gamma_{0}^{2} \frac{v^{2}}{c^{2}} \pi_{m}-\frac{\gamma_{0}^{2}}{c^{2}}\left[(\bar{P} \cdot \bar{v})(\bar{E} \cdot \bar{v})+\left(\mu_{0} \bar{M} \cdot \bar{v}\right)(\bar{H} \cdot \bar{v})\right]
\end{aligned}
$$

Carrying out the operations implied in (90) to obtain the force density, one finds

$$
\begin{aligned}
\overline{\mathrm{f}}_{\mathrm{k}}= & -\nabla \pi_{\mathrm{m}}+(\overline{\mathrm{P}} \cdot \nabla) \overline{\mathrm{E}}+\left(\mu_{0} \overline{\mathrm{M}} \cdot \nabla\right) \overline{\mathrm{H}} \\
& +\overline{\mathrm{v}} \times(\overline{\mathrm{P}} \cdot \nabla) \mu_{0} \overline{\mathrm{H}}-\overline{\mathrm{v}} \times\left(\mu_{0} \overline{\mathrm{M}} \cdot \nabla\right) \epsilon_{0} \overline{\mathrm{E}} \\
& +\left[\frac{\partial}{\partial \mathrm{t}} \overline{\mathrm{P}}+\nabla \cdot(\overline{\mathrm{v}} \overline{\mathrm{P}})\right] \times \mu_{0} \overline{\mathrm{H}} \\
& -\left[\frac{\partial}{\partial \mathrm{t}} \mu_{0} \overline{\mathrm{M}}+\nabla \cdot\left(\overline{\mathrm{v}} \mu_{0} \overline{\mathrm{M}}\right)\right] \times \epsilon_{0} \overline{\mathrm{E}} \\
& -\frac{\partial}{\partial t} \bar{G}_{\mathrm{m}}-\nabla \cdot\left(\overline{\mathrm{v} \bar{G}_{\mathrm{m}}}\right) .
\end{aligned}
$$

In a similar way one finds that

$$
\phi_{\mathrm{k}}=\overline{\mathrm{f}}_{\mathrm{k}} \cdot \overline{\mathrm{v}} .
$$

A remarkable feature about the expressions for force, pressure, stress, and momentum in the $\mathrm{E}-\mathrm{H}$ formulation is that they can be interpreted easily by means of a 
reasonable model for a polarizable and magnetizable fluid. Because this model gives good physical insight, we develop it in Section XI.

We have used the Minkowski and E-H formulations to predict the force density $\bar{f}_{k}$. The expressions, (82) and (107), look different formally, yet it is shown in the Appendix that they are, in fact, identical. 


\section{INTERPRETATION OF THE PRESSURE}

The pressure, stress, momentum, and force predicted in Section IX can be interpreted in terms of a simple model for polarization and magnetization. In this section we discuss the pressure $\pi_{m}$, without relying upon the model. Then in Section XI, we interpret the stress, momentum, and force by means of a dipole model for $\overline{\mathrm{P}}$ and $\overline{\mathrm{M}}$.

The pressure $\pi_{\mathrm{m}}$ was introduced in (101) in order to account for changes in energy per particle caused by changes in density. If the various particles do not interact except through macroscopic fields, then the energy of each particle (for the same polarization per particle $\overline{\mathrm{P}} / \mathrm{n}$ and magnetization per particle $\overline{\mathrm{M}} / \mathrm{n}$ ) should not depend on whether or not other particles are nearby or how many are nearby. Thus we have established the general principle that for noninteracting particles, $\pi_{m}=0$. This is true regardless of the constitutive laws of the individual particles and holds for a wide class of material, including time-dispersive, gyromagnetic, and gyroelectric media. Thus the pressure $\pi_{m}$ accounts for interaction of particles.

For the next example for calculation of $\pi m$, suppose that the rest-frame energy density $\mathrm{W}_{\mathrm{m}}^{0}$ is known to be a function of $\overline{\mathrm{P}}^{0}, \overline{\mathrm{M}}^{0}$, and $\mathrm{n}^{0}$ for adiabatic processes. It is equivalent to say that $\mathrm{W}_{\mathrm{m}}^{0} / \mathrm{n}^{0}$ is a function of $\overline{\mathrm{P}}^{0} / \mathrm{n}^{0}, \overline{\mathrm{M}}^{0} / \mathrm{n}^{0}$, and $\mathrm{n}^{0}$. Then

$$
\frac{\partial}{\partial t}\left(\frac{W_{m}^{0}}{n^{0}}\right)=\frac{\partial\left(W_{m}^{0} / n^{0}\right)}{\partial\left(\bar{P}^{0} / n^{0}\right)} \cdot \frac{\partial\left(\bar{P}^{0} / n^{0}\right)}{\partial t}+\frac{\partial\left(W_{m}^{0} / n^{0}\right)}{\partial\left(\mu_{0} \bar{M}^{0} / n^{0}\right)} \cdot \frac{\partial\left(\mu_{0} \bar{M}^{0} / n^{0}\right)}{\partial t}+\frac{\partial\left(w_{m}^{0} / n^{0}\right)}{\partial n^{0}} \frac{\partial n^{0}}{\partial t}
$$

and comparison with (101) shows that the three partial derivatives of $\mathrm{w}_{\mathrm{m}}^{0} / \mathrm{n}^{0}$ are, respectively, $\overline{\mathrm{E}}^{0}, \overline{\mathrm{H}}^{0}$, and $\pi_{\mathrm{m}} /\left(\mathrm{n}^{0}\right)^{2}$. But since $\mathrm{W}_{\mathrm{m}}^{0} / \mathrm{n}^{0}$ is a function of the three variables, second cross-partial derivatives are equal in pairs. Consequently we can find the derivatives of $\pi_{m}$ with respect to $\overline{\mathrm{P}}^{0}$ and $\overline{\mathrm{M}}^{0}$ in terms of the constitutive relations between the electromagnetic variables. Thus $\pi_{\mathrm{m}}$ is of the form

$$
\pi_{m}=\pi_{h}\left(n^{0}\right)+\left(n^{0}\right)^{2} \int_{0}^{\bar{P}^{0} / n^{0}} \frac{\partial \bar{E}^{0}}{\partial n^{0}} \cdot d\left(\bar{P}^{0} / n^{0}\right)+\left(n^{0}\right)^{2} \int_{0}^{\mu_{0} \bar{M}^{0} / n^{0}} \frac{\partial \bar{H}^{0}}{\partial n^{0}} \cdot d\left(\mu_{0} \bar{M}^{0} / n^{0}\right)
$$

where the partial derivatives are evaluated for constant $\overline{\mathrm{P}}^{0} / \mathrm{n}^{0}$ and $\overline{\mathrm{M}}^{0} / \mathrm{n}^{0}$, the integrals are evaluated for the final value of $n^{0}$, and $\pi_{h}\left(n^{0}\right)$, which is the pressure for zerofields, is a function of only $n^{0}$. This formula (110) is for the case for which the constitutive laws relating $\overline{\mathrm{P}}^{0}$ to $\overline{\mathrm{E}}^{0}$ and $\mathrm{n}^{0}$ do not involve $\overline{\mathrm{H}}^{0}$, and the laws relating $\overline{\mathrm{M}}^{0}$ to $\overline{\mathrm{H}}^{0}$ and $\mathrm{n}^{0}$ do not involve $\overline{\mathbf{E}}^{0}$. This formula can be put in a variety of additional forms, including

$$
\pi_{m}=\pi_{h}\left(n^{0}\right)-\left(n^{0}\right)^{2} \int_{0}^{\bar{E}^{0}} \frac{\partial\left(\bar{P}^{0} / n^{0}\right)}{\partial n^{0}} \cdot d \bar{E}^{0}-\left(n^{0}\right)^{2} \int_{0}^{\bar{H}^{0}} \frac{\partial\left(\mu_{0} \bar{M}^{0} / n^{0}\right)}{\partial n^{0}} \cdot d \bar{H}^{0}
$$


where $\overline{\mathrm{E}}^{0}$ and $\overline{\mathrm{H}}^{0}$ are held fixed in the derivatives, and

$$
\begin{aligned}
\pi_{m}= & \pi_{h}\left(n^{0}\right)+\bar{E}^{0} \cdot \overline{\mathrm{P}}^{0}+\overline{\mathrm{H}}^{0} \cdot \mu_{0} \overline{\mathrm{M}}^{0}+\int_{0}^{\overline{\mathrm{P}}^{0}}\left(\mathrm{n}^{0} \frac{\partial \overline{\mathrm{E}}^{0}}{\partial \mathrm{n}^{0}}-\overline{\mathrm{E}}^{0}\right) \cdot \mathrm{d} \overline{\mathrm{P}}^{0} \\
& +\int_{0}^{\mu_{0} \overline{\mathrm{M}}^{0}}\left(\mathrm{n}^{0} \frac{\partial \overline{\mathrm{H}}^{0}}{\partial \mathrm{n}}-\overline{\mathrm{H}}^{0}\right) \cdot \mathrm{d} \mu_{0} \overline{\mathrm{M}}^{0}
\end{aligned}
$$

where $\overline{\mathrm{P}}^{0}$ and $\overline{\mathrm{M}}^{0}$ are held constant in the derivatives.

For a linear medium satisfying the relations,

$$
\begin{aligned}
& \left.\overline{\mathrm{P}}^{0}=\epsilon_{0} \mathrm{X}_{\mathrm{e}} \mathrm{(n}^{0}\right) \overline{\mathrm{E}}^{0} \\
& \overline{\mathrm{M}}^{0}=\chi_{\mathrm{m}}\left(\mathrm{n}^{0}\right) \overline{\mathrm{H}}^{0},
\end{aligned}
$$

$\pi_{\mathrm{m}}$ reduces to

$$
\pi_{m}=\pi_{h}\left(n^{0}\right)+\frac{1}{2} \epsilon_{0}\left(\overline{\mathrm{E}}^{0}\right)^{2}\left(x_{e}-n^{0} \frac{\partial x_{e}}{\partial n^{0}}\right)+\frac{1}{2} \mu_{0}\left(\bar{H}^{0}\right)^{2}\left(x_{m}-n^{0} \frac{\partial x_{m}}{\partial n^{0}}\right),
$$

a form that is in agreement with results on stationary media. ${ }^{18}$ Furthermore, if the particular dependence of $x_{e}$ and $x_{m}$ is given by the Clausius-Mossotti relations 19,20

$$
\pi_{m}=\pi_{h}\left(n^{0}\right)-\frac{\left(\overline{\mathrm{P}}^{0}\right)^{2}}{6 \epsilon_{0}}-\frac{\mu_{0}}{6}\left(\overline{\mathrm{M}}^{0}\right)^{2} \text {. }
$$

For more complicated constitutive laws the expression for $\pi_{\mathrm{m}}$ would be different. In all cases, however, $\pi_{m}$ accounts for particle interactions other than those through macroscopic fields, and generally includes the hydrodynamic pressure, if any. 


\section{MODEL OF A POLARIZABLE FLUID}

In this section we shall discuss a model $^{21}$ for polarization for which it is possible to derive the force density without recourse to thermodynamics. We shall limit our discussion to a polarizable fluid and shall not consider magnetization in detail because the extension to this case is exactly analogous. We shall consider a fluid consisting of noninteracting electric dipoles each of vector strength

$$
\overline{\mathrm{p}}=q \overline{\mathrm{d}}
$$

where $\mathrm{q}$ is the magnitude of the charges, and $\overline{\mathrm{d}}$ is the vector distance between them.

(Compare Section VI.) We interpret the dipole strength (118) as that observed in the laboratory frame in which the dipole may possess an arbitrary velocity $\bar{v}$. We assign to the negative charge portion of the dipole, the velocity $\bar{v}(\bar{r})$. The positive charge of the dipole then possesses the velocity

$$
\bar{v}_{+}(\bar{r})=\bar{v}(\bar{r})+\frac{d \bar{d}}{d t} .
$$

The forces acting on the dipoles are assumed to be due to long-range forces only. Thus a fluid of this kind cannot exhibit pressure or electrostrictive effects and we cannot expect to reproduce the term $\nabla_{\pi_{m}}$ in (107).

The net force acting on a dipole as evaluated in the laboratory frame is obtained as the sum of the force acting on the + charge of the dipole and that acting on the - charge. In this connection one must take into account the possible variations of the electric and magnetic fields over the distance $\bar{d}$. One obtains, to first order in $\bar{d}$,

$$
\begin{aligned}
\overline{\mathrm{F}}_{\mathrm{d}} & =\mathrm{q}\left[\overline{\mathrm{E}}(\overline{\mathrm{r}}+\overline{\mathrm{d}})+\overline{\mathrm{v}}_{+}(\overline{\mathrm{r}}) \times \mu_{0} \overline{\mathrm{H}}(\overline{\mathrm{r}}+\overline{\mathrm{d}})\right]-\mathrm{q}\left[\overline{\mathrm{E}}(\overline{\mathrm{r}})+\overline{\mathrm{v}}(\overline{\mathrm{r}}) \times \mu_{0} \overline{\mathrm{H}}(\overline{\mathrm{r}})\right] \\
& =\mathrm{q} \overline{\mathrm{d}} \cdot \nabla \overline{\mathrm{E}}+\mathrm{q} \overline{\mathrm{v}} \times \mu_{0}(\overline{\mathrm{d}} \cdot \nabla) \overline{\mathrm{H}}+\mathrm{q} \frac{\mathrm{d} \overline{\mathrm{d}}}{\mathrm{dt}} \times \mu_{0} \overline{\mathrm{H}} .
\end{aligned}
$$

The polarization density $\overline{\mathrm{P}}$ of the dielectric fluid is obtained as the dipole strength per unit volume. If $\mathrm{N}$ is the number of dipoles per unit volume, one has

$$
\overline{\mathrm{P}}=\mathrm{Nq} \overline{\mathrm{d}} \text {. }
$$

The expression $\bar{F}_{\mathrm{d}}$ of (120) can be converted into a force density by adding the forces acting on all dipoles per unit volume

$$
\overline{\mathrm{f}}_{\mathrm{d}}=N \overline{\mathrm{F}}_{\mathrm{d}}=\overline{\mathrm{P}} \cdot \nabla \overline{\mathrm{E}}+\overline{\mathrm{v}} \times \mu_{0}(\overline{\mathrm{P}} \cdot \nabla) \overline{\mathrm{H}}+\left[\frac{\partial \overline{\mathrm{P}}}{\partial \mathrm{t}}+\nabla \cdot(\overline{\mathrm{v}} \overline{\mathrm{P}})\right] \times \mu_{0} \overline{\mathrm{H}} .
$$

Here we have used the continuity law satisfied by the dipole number density

$$
\frac{\partial N}{\partial t}+\nabla \cdot(\bar{v} N)=0 \text {. }
$$

Equation(122) gives the force per unit volume acting on the dipoles in the dielectric fluid. 


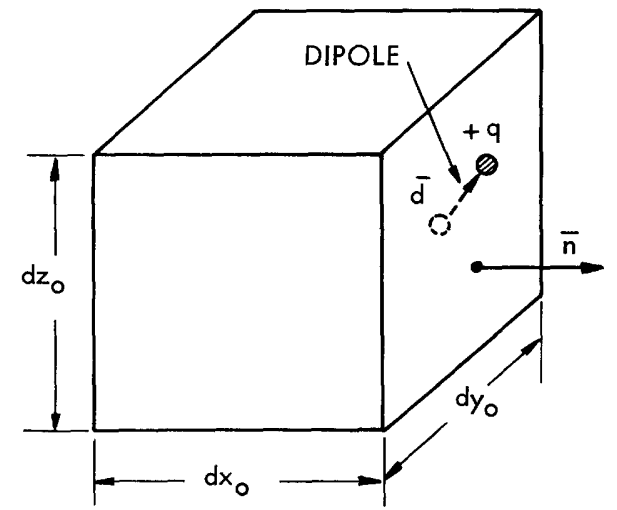

Fig. 2. A volume $\mathrm{V}$ with outward-directed normal $\bar{n}$ on its surface.
Not all of this force density is utilized in the acceleration of the fluid mass density. The binding forces between the + and - charges store energy. Energy in motion acquires momentum according to the relativistic transformation laws. Furthermore, a stress is introduced on a volume element $\mathrm{dV}$ containing the mass $\rho \mathrm{dV}$. Indeed, if one draws the boundaries of this volume element in the rest frame as shown in Fig. 2, one finds that the binding forces of all those positive charges outside the element whose neg-

ative partners are inside the volume element, pass through the boundary. The net force passing the boundary $\mathrm{dy}_{0} \mathrm{dz}_{0}$ is equal to the product of the force $\left(\mathrm{q}_{\mathrm{x}} \overline{\mathrm{x}}^{0}\right.$ ) times the number of dipoles piercing this boundary $\left(\mathrm{N}_{0} \mathrm{dy}_{0} \mathrm{dz}{ }_{0}\right)$. One has for the net force per unit area

$$
\overline{\mathrm{n}} \cdot \overline{\mathrm{P}}^{0} \overline{\mathrm{E}}^{0}
$$

where $\bar{n}$ is the unit vector normal to the element $d_{y_{0}} \mathrm{dz}_{0}$. In this way one concludes that the polarization of the fluid has an associated stress tensor $-\overline{\mathrm{P}}^{0} \overline{\mathrm{E}}^{0}$. Furthermore, it has the energy density $W_{m}^{0}$. Stress and energy densities in motion possess momentum relativistically. The force density $\bar{f}_{d}$ of (122) must overcome this momentum as well as the inertia associated with the mass of the fluid. The momentum associated with the stress and energy density may be found from the results of the preceding section as $\bar{G}_{m}$. The force density $\bar{f}_{k}$ producing the acceleration of the fluid is that portion of $\bar{f}_{d}$ that remains after the force density responsible for the total rate of change of the momentum has been subtracted.

$$
\begin{aligned}
\bar{f}_{k} & =\bar{f}_{d}-\frac{\partial}{\partial t} \bar{G}_{m}-\nabla \cdot\left(\bar{v}_{\mathrm{G}_{m}} \bar{y}^{\prime}\right. \\
& =\bar{P} \cdot \nabla \bar{E}+\bar{v} \times \mu_{0}(\bar{P} \cdot \nabla) \bar{H}+\left[\frac{\partial \bar{P}}{\partial t}+\nabla \cdot(\bar{v} \bar{P})\right] \times \mu_{0} \bar{H}-\frac{\partial}{\partial t} \bar{G}_{m}-\nabla \cdot\left(\bar{v} \bar{G}_{m}\right) .
\end{aligned}
$$

This equation checks with (107) if magnetization effects are omitted and $\pi_{m}$ is set equal to zero. The generalization to a fluid exhibiting magnetization is easy.

In this way we have found a simple interpretation for most of (107). Pressure effects and electrostrictive effects can be included phenomenologically by assigning instead of the tensor (124) a new stress tensor in the rest frame, $\pi_{m} \overline{\bar{\delta}}-\overline{\mathrm{P}}^{0} \overline{\mathrm{E}}^{0}$. The inclusion of $\pi_{m}$ has two obvious consequences. First of all, it contributes through its gradient to the force density; second, it contributes to the momentum associated with the stress tensor in a moving fluid. This is exactly the way in which the inclusion of pressure affects (107).

We have therefore interpreted every term in (107). 


\section{APPENDIX}

We have used both the Minkowski and E-H formulations to derive expressions for $\overline{\mathrm{f}_{\mathrm{k}}}$, (82) and (107). These appear to be different, and we now wish to prove that the two are actually the same. Since in the Minkowski formulation

$$
\overline{\mathrm{f}}_{\mathrm{k}}=-\nabla \cdot \overline{\overline{\mathrm{T}}}_{\mathrm{M}}-\frac{\partial \overline{\mathrm{G}}_{\mathrm{M}}}{\partial \mathrm{t}}
$$

and in the $\mathrm{E}-\mathrm{H}$ formulation

$$
\overline{\mathrm{f}}_{\mathrm{k}}=-\nabla \cdot\left(\overline{\overline{\mathrm{T}}}_{\mathrm{e}}+\overline{\overline{\mathrm{T}}}_{\mathrm{m}}\right)-\frac{\partial}{\partial \mathrm{t}}\left(\overline{\mathrm{G}}_{\mathrm{e}}+\overline{\mathrm{G}}_{\mathrm{m}}\right)
$$

we need only prove that

$$
\overline{\overline{\mathrm{T}}}_{\mathrm{M}}=\overline{\overline{\mathrm{T}}}_{\mathrm{e}}+\overline{\overline{\mathrm{T}}}_{\mathrm{m}}
$$

and

$$
\bar{G}_{M}=\bar{G}_{e}+\bar{G}_{m}
$$

But each of these quantities is related to corresponding quantities in the rest frame by means of the transformation laws (55), and hence it is sufficient to show

$$
\begin{aligned}
& \overline{\overline{\mathrm{T}}}_{\mathrm{M}}^{0}=\overline{\overline{\mathrm{T}}}_{\mathrm{e}}^{0}+\overline{\overline{\mathrm{T}}}_{\mathrm{m}}^{0} \\
& \overline{\mathrm{G}}_{\mathrm{M}}^{0}=\overline{\mathrm{G}}_{\mathrm{e}}^{0}+\overline{\mathrm{G}}_{\mathrm{m}}^{0} \\
& \overline{\mathrm{S}}_{\mathrm{M}}^{0}=\overline{\mathrm{S}}_{\mathrm{e}}^{0}+\overline{\mathrm{S}}_{\mathrm{m}}^{0} \\
& \mathrm{~W}_{\mathrm{M}}^{0}=\mathrm{w}_{\mathrm{e}}^{0}+\mathrm{w}_{\mathrm{m}}^{0} .
\end{aligned}
$$

To facilitate comparisons, we note from (7) that in the rest frame the relations between the field variables in the two formulations is particularly simple: $\overline{\mathrm{E}}^{0}$ and $\overline{\mathrm{H}}^{0}$ are the same in the two formulations, and $\overline{\mathrm{D}}^{0}$ and $\overline{\mathrm{B}}^{0}$ of the Minkowski formulation are equal, respectively, to $\epsilon_{0} \overline{\mathrm{E}}^{0}+\overline{\mathrm{P}}^{0}$ and $\mu_{0}\left(\overline{\mathrm{H}}^{0}+\overline{\mathrm{M}}^{0}\right)$ in the $\mathrm{E}-\mathrm{H}$ formulation. We can easily verify (A. 6) by use of (79), (20), and (105) and verify (A. 7) by use of (72), (21), and (100).

It is more difficult to prove (A. 8). In the absence of electromagnetic fields, (A. 8) is certainly satisfied. However, the energies are not measurable, and what we really should prove is that for equivalent constitutive relations, (A. 8) is satisfied. Consider first the case with no magnetic fields. If the constitutive relations for the electric-field quantities are that $\overline{\mathrm{D}}^{0}$ and $\overline{\mathrm{P}}^{0}$ are functions of $\overline{\mathrm{E}}^{0}$ and $\mathrm{n}^{0}$, then from (69)

$$
w_{M}^{0}=w_{M}^{0}\left(\bar{D}^{0}=0\right)+\int_{0}^{\bar{D}^{0}} \bar{E}^{0} \cdot d \bar{D}^{0}
$$


whereas from (22) and (101),

$$
\mathrm{w}_{\mathrm{m}}^{0}+\mathrm{w}_{\mathrm{e}}^{0}=\mathrm{w}_{\mathrm{m}}^{0}\left(\overline{\mathrm{P}}^{0}=0\right)+\frac{1}{2} \epsilon_{0}\left(\overline{\mathrm{E}}^{0}\right)^{2}+\int_{0}^{\overline{\mathrm{P}}^{0}} \overline{\mathrm{E}}^{0} \cdot \mathrm{d} \overline{\mathrm{P}}^{0} .
$$

Because $\bar{D}^{0}=\epsilon_{0} \overline{\mathrm{E}}^{0}+\overline{\mathrm{P}}^{0}$ these two expressions are equal. Similar arguments hold if magnetic fields are present.

Our only remaining task is to demonstrate (A. 5). From (78), (19), and (104), we see that (A. 5) holds provided

$$
\pi_{M}+\frac{1}{2} \overline{\mathrm{E}}^{0} \cdot \overline{\mathrm{D}}^{0}+\frac{1}{2} \overline{\mathrm{H}}^{0} \cdot \overline{\mathrm{B}}^{0}=\pi_{\mathrm{m}}+\frac{1}{2} \epsilon_{0}\left(\overline{\mathrm{E}}^{0}\right)^{2}+\frac{1}{2} \mu_{0}\left(\overline{\mathrm{H}}^{0}\right)^{2} .
$$

This fact is most easily shown from (76) on the one hand, and (101) and (22) on the other hand, making use of (A. 8). 


\section{References}

1. R. M. Fano, L. J. Chu, and R. B. Adler, Electromagnetic Fields, Energy, and Forces (John Wiley and Sons, Inc., New York, 1960), Section A 1.5.1.

2. C. T. Tai, A study of electrodynamics of moving media, Proc. IEEE 52, 685689 (1964).

3. P. Penfield, Jr., and C. T. Tai, Electromagnetism of Moving Media, Proc. IEEE 52, $1361-1362$ (1964).

4. C. M $\phi 1 l e r$, The Theory of Relativity (Oxford University Press, 1952; reprinted 1955).

5. Ibid., see Sec. 50.

6. Ibid., see Sec. 75.

7. B. de Sa, Der Energie-Impuls-Tensor in der Relativistischen Thermodynamik Irreversibler Prozesse, Dr. Nat. Thesis, T. H. Aachen, Germany, February 9, 1960.

8. C. L. Tang and J. Meixner, Relativistic Theory of the Propagation of Plane Electromagnetic Waves, Phys. Fluids 4, 148-154 (1961).

9. J. Meixner, Relativistic Thermodynamics of Irreversible Processes in a One Component Fluid in the Presence of Electromagnetic Fields, Report RL-184, Radiation Laboratory, University of Michigan, April 1961.

10. J. Meixner, Der Drehimpulssatz in der Thermodynamik der irreversiblen Prozesse, Z. Physik 164, 145-155 (1961).

11. C. Mфller, op. cit., Sec. 17.

12. R. M. Fano, L. J. Chu, and R. B. Adler, op. cit., see Appendix A.

13. C. Mфller, op. cit., Sec. 21.

14. J. A. Stratton, Electromagnetic Theory (McGraw-Hill Book Company, Inc., New York, 1941), Sec. 1.23.

15. R. M. Fano, L. J. Chu, and R. B. Adler, op. cit., Sec. A 1.5.

16. J. Meixner, Thermodynamik in Gegenwart Elektromagnetischer Felder, Int. J. Engrg. Sci. 1, 177-186 (1963).

17. L. D. Landau and E. M. Lifshitz, Electrodynamics of Continuous Media (Pergamon Press, Oxford, and Addison-Wesley Publishing Company, Inc., Reading, Mass., 1960).

18. W. F. Brown, Jr., Electric and Magnetic Forces: A Direct Calculation, Am. J. Phys. 19, 299-304 (1951); see Sec. 2.3.

19. J. A. Stratton, op. cit., p. 140.

20. W. K. H. Panofsky and M. Phillips, Classical Electricity and Magnetism (Addison-Wesley Publishing Company, Inc., Reading, Mass., 1955), Sec. 2.4.

21. R. M. Fano, L. J. Chu, and R. B. Adler, op. cit., Sec. 5.2. 


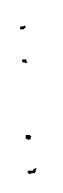




\section{Department of Defense}

Dr Edward M. Reilley

Asst Director (Research)

Ofc of Defense Res \& Eng

Department of Defense

Washington, D.C. 20301

Dr James A. Ward

Office of Deputy Director (Research

and Information Rm 3D1037

Department of Defense

The Pentagon

Washington, D.C. 20301

Director

Advanced Research Projects Agency

Department of Defense

Washington, D.C. 20301

Mr Charles Yost, Director

For Materials Sciences

Advanced Research Projects Agency

Department of Defense

Washington, D.C. 20301

Defense Documentation Center

Attn: TISIA

Cameron Station, Bldg 5

Alexandria, Virginia 22314

Director

National Security Agency

Attn: C3/TDL

Fort George G. Meade, Maryland 20755

\section{Department of the Army}

Chief of Research and Development Headquarters, Department of the Army Attn: Physical Sciences Division P\&E Washington, D.C. 20310

Research Plans Office

U.S. Army Research Office

3045 Columbia Pike

Arlington, Virginia 22204

Commanding Officer

Foreign Service \& Technology Center

Arlington Hall

Arlington, Virginia

Commanding General

U.S. Army Materiel Command

Attn: AMCRD-RS-PE-E

Washington, D.C. 20315
Commanding General

U.S. Army Strategic Communications Command

Washington, D.C. 20315

Commanding General

U.S. A rmy Materials Research A gency

Watertown Arsenal

Watertown, Massachusetts 02172

Commanding Officer

U.S. A rmy Ballistics Research Laboratory

Attn: V.W. Richards

Aberdeen Proving Ground

Aberdeen, Maryland 21005

Commandant

U.S. Army Air Defense School

Attn: Missile Sciences Division, C\&S Dept.

P.O. Box 9390

Fort Bliss, Texas 79916

Commanding General

U.S. Army Missile Command

Attn: Technical Library

Redstone Arsenal, Alabama 35809

Commanding General

Frankford Arsenal

Attn: SMUFA - 1310 (Dr Sidney Ross)

Philadelphia, Pennsylvania 19137

U.S. Army Munitions Command

Attn: Technical Information Branch

Picatinney Arsenal

Dover, New Jersey 07801

Commanding Officer

Harry Diamond Laboratories

Attn: Mr Berthold Altman

Connecticut Avenue and Van Ness Street N.W.

Washington, D.C. 20438

Commanding Officer

Harry Diamond Laboratories

Attn: Dr R.T. Young

Electron Tubes Division

Connecticut Avenue and Van Ness Street N.W.

Washington, D.C. 20438

Commanding Officer

U.S. Army Security A gency

Arlington Hall

A rlington, Virginia 22212

Commanding Officer

U.S. Limited War Laboratory

Attn: Technical Director

Aberdeen Proving Ground

Aberdeen, Maryland 21005 
Commanding Officer

Human Engineering Laboratories

Aberdeen Proving Ground

Maryland 21005

Director

U.S. Army Engineer Geodesy, Intelligence and Mapping

Research and Development Agency

Fort Belvoir, Virginia 22060

Commandant

U.S. A rmy Command and General Staff College

Attn: Secretary

Fort Leavenworth, Kansas 66207

Dr. H. Robl, Deputy Director

U.S. A rmy Research Office (Durham)

P.O. Box CM, Duke Station

Durham, North Carolina 27706

Commanding Officer

U.S. Army Research Office (Durham)

Attn: CRD-AA-IP (Richard O. Ulsh)

P.O. Box CM, Duke Station

Durham, North Carolina 27706

Commanding General

U.S. Army Electronics Command

Attn: AMSEL-SC

Fort Monmouth, New Jersey 07703

Director

U.S. Army Electronics Laboratories

Attn: Dr S. Benedict Levin, Director

Institute for Exploratory Research

Fort Monmouth, New Jersey 07703

Director

U.S. A rmy Electronics Laboratories

Attn: Mr Robert O. Parker, Executive Secretary JSTAC (AMSEL-RD-X)

Fort Monmouth, New Jersey 07703

Superintendent

U.S. Army Military Academy

West Point, New York 10996

The Walter Reed Institute of Research Walter Reed Army Medical Center

Washington, D.C. 20012

Director

U.S. A rmy Electronics Laboratories

Fort Monmouth, New Jersey 07703

Attn: AMSEL-RD-DR NE SS

$\begin{array}{lll}\text { X } & \text { NO } & \text { PE } \\ \text { XE } & \text { NP } & \text { PR } \\ \text { XC } & \text { SA } & \text { PF } \\ \text { XS } & \text { SE } & \text { GF } \\ \text { NR } & \text { SR } & \text { ADT } \\ & & \text { FU\#1 }\end{array}$

Commanding Officer

U.S. Army Electronics R\&D Activity

Fort Huachuca, Arizona 85163

Commanding Officer

U.S. Army Engineers R\&D Laboratory

Attn: STINFO Branch

Fort Belvoir, Virginia 22060

Commanding Officer

U.S. Army Electronics R\&D Activity

White Sands Missile Range

New Mexico 88002

Director

Human Resources Research Office

The George Washington University

$300 \mathrm{~N}$. Washington Street

Alexandria, Virginia 22314

Commanding Officer

U.S. Army Personnel Research Office

Washington, D.C.

Commanding Officer

U.S. Army Medical Research Laboratory

Fort Knox, Kentucky

Department of the Air Force

Director

Air University Library

Maxwell A.F. Base, Alabama

Commander

Air Force Office of Scientific Research

Washington 25, D.C.

Attn:-SREE

Department of The Air Force

Headquarters-United States Air Force

Washington $25, \mathrm{D}$. C.

Attn: AFTAC/TD-1

Dr. Harvey E. Savely, SRL

Air Force Office of Sci. Res.

Office of Aerospace Research, USAF

Washington 25, D. C.

Mr. C.N. Hasert

Scientific Advisory Board

$\mathrm{Hq}$, USA F

Washington 25, D.C. 


\section{JOINT SERVICES DISTRIBUTION LIST (continued)}

APGC (PGBA P- 1)

Elgin Air Force Base

Florida 32542

AFETR

(AFETR Tech. Library MU-135)

Patrick Air Force Base

Cocoa, Florida

Air Force Cambridge Res. Lab. L. G. Hanscom Field

Bedford, Massachusetts 01731

Attn: CRDM, Mr. Herskovitz

Commander, AFCRL

Attn: C. P. Smith (CRBS)

L. G. Hanscom Field

Bedford, Massachusetts

Dr. L. C. Block

AFCRL (CROV)

L. G. Hanscom Field

Bedford, Massachusetts

AFCRL

Office of Aerospace Res., USAF

Bedford, Mass.

Attn: CRDA

Mr. Rocco H. Urbano, Chief AFCRL, A ppl. Math. Branch

Data Sciences Laboratory

Laurence G. Hanscom Field

Bedford, Massachusetts

AFCRL (CRFE-Dr. Nicholas Yannoni)

L. G. Hanscom Field

Bedford, Massachusetts

S. H. Sternick

Aerospace Comm. - Attn: ESNC

Waltham Federal Center

424 Trapelo Road

Waltham, Massachusetts 02154

Rome Air Dev. Center (RAWL, H. Webb)

Griffiss Air Force Base

New York 13442

Systems Engineering Group

Deputy for Systems Eng'g., SEPRR

Directorate of Tech. Pubs. and Specs.

Wright-Patterson AFB, OHIO 45433

Aeronautical Systems Division

Attn: ASRPE, Mr. Robt: Cooper

Wright-Patters on AFC, Ohio 45433
Aeronautical Systems Division

Attn: ASRPP-20 (Mr. Don R. Warnock)

Wright-Patterson AFB, Ohio 45433

AFAL

AVR (L)

Wright-Patterson AFB

Ohio 45433

Dr. H. H. Kurzweg

Director Research - OART

NASA

Washington, D.C. 20546

Systems Engineering Group (RTD)

Attn: SEPIR

Wright-Patterson AFB, Ohio 45433

AFAL (AVTE)

Wright-Patterson AFB

Ohio 45433

Mr. Roland Chase

National Aeronautics \& Space Administration

1512 H Street, N.W.

Washington 25, D.C.

Professor A rwin Dougal

University of Texas

EE Department

Austin, Texas

Honorable Alexander H. Flax

Asst Secretary of the Air Force (R\&D)

Office of the Secretary of the Air Force

Washington 25, D. C.

Professor Nicholas George

California Institute of Technology

EE Department

Pasadena, California

Dr. Lowell M. Hollingsworth

AFCRL

L.G. Hanscom Field

Bedford, Massachusetts

Dr. Zohrab Kaprielian

University of Southern California

University Park

Los Angeles 7, California 
Dr. John M. Ide

National Science Foundation

Washington 25, D. C.

Lt Col Edwin M. Myers

Headquarters USAF (AFRDR)

Washington 25, D. C.

Professor Wm. H. Radford

Director, Lincoln Laboratories

Lexington, Massachusetts

Brig Gen B. G. Holzman, USAF (Ret.)

Electronics Research Center, NASA

30 Memorial Drive

Cambridge, Mass.

Dr. R. L. Sproull

Director, Advanced Research Projects A gency

Washington 25, D. C.

Brigadier General J. T. Stewart Director of Science \& Technology Deputy Chief of Staff (R\&D)

USA F

Washington 25, D. C.

Mr. James Tippett

National Security Agency

Fort Meade, Maryland

Dr. H. Harrison

NASA (Code RRE)

Fourth and Independence Streets

Washington, D.C. 20546

AEC

Civ of Tech Info Ext

P.O. Box 62

Oak Ridge, Tenn.

AFRST (SC/EN)

Lt Col L. Stone

Rm 4C 341

The Pentagon

Washington, D.C. 20301

U.S. Atomic Energy Commission Library

Gaithersburg, Md. 20760
ARL (ARD/Col R.E. Fontana)

Wright-Patterson AFB,

Ohio 45433

Office of Research Analyses

Attn: Col K.W. Gallup

Holloman AFB, NMex 88330

AFCRL (CRXL)

L. G. Hanscom Fld

Bedford, Mass 01731

Frank J. Seiler Rsch Lab

Library

USA F A cademy, Colo 80840

ARL (AROL)

Wright-Patterson AFB,

Ohio 45433

Office of Research Analyses

Library

Holloman AFB, NMex 88330

LOOAR (Library)

AF Unit Post Office

Los Angeles, Calif 90045

Churchill Research Range

Library

Fort Churchill

Manitoba, Canada

Los Alamos Scientific Lab

Attn: Technical Library

Los Alamos, NMex 87544

Battelle Memorial Institute

Technical Library

$505 \mathrm{King}$ Avenue

Columbus, Ohio 43201

John Crerar Library

35 West 33rd St.

Chicago, Ill.

Linda Hall Library

5109 Cherry St.

Kansas City, Mo.

National Science Foundation Library

1951 Constitution Ave., N.W.

Washington, D.C. 20550 
Johns Hopkins University

A pplied Physics Lab Library

White Oak

Silver Spring, Md. 20910

Stanford Research Institute

Library

820 Mission St.

South Pasadena, Calif. 91030

Southwest Research Institute

Library

8500 Culebra Road

San Antonio, Texas

ARPA, Tech Info Office

The Pentagon

Washington, D.C. 20301

DDR\&E (Tech Library)

Rm 3C 128

The Pentagon

Washington, D.C. 20301

Industrial College of the

Armed Forces

Attn: Library

Washington, D.C.

AFIT (MCLI)

Tech Library

Wright-Patterson AFB

Ohio 45433

AUL 3T-9663

Maxwell AFB, Ala 36112

USAFA (DLIB)

USAF A cademy, Colorado 80840

AFSC (Tech Library)

Andrews AFB

Washington, D.C. 20331

ASD (Tech Library)

Wright-Patterson, AFB

Ohio 45433

BSD (Tech Library)

Norton AFB, Calif 92409

ESD (ESTI)

L. G. Hanscom Field, F172

Bedford, Mass 01731
RTD (Tech Library)

Bolling AFC, D. C. 20332

AFFTC (Tech Library)

Edwards AFB, Calif 93523

AFMDC (Tech Library)

Holloman AFB, NMex 88330

AFWL (WLIL, Tech Library)

Kirtland AFB, NMex 87117

A PGC (Tech Library)

Eglin AFB, Fla 32542

AEDC (Tech Library)

Arnold AFS, Tenn 37389

RADC (Tech Library)

Griffiss AFB, N.Y. 13442

Director

National Aeronautical Establishment

Ottawa, Ontario, Canada

CIA

OCR/LY/IAS

IH $129 \mathrm{Hq}$

Washington, D.C. 20505

National Defense Library

Headquarters

Ottawa, Ontario, Canada

Technical Library

White Sands Missile Range

NMex 88002

NASA/AFSS/ 1 FOB6

Tech Library, Rm 60084

Washington, D.C. 20546

Space Systems Division

Los Angeles Air Force Station

Air Force Unit Post Office

Los Angeles, California 90045

Attn: SSSD

U.S. Regional Science Office/LAOAR

U.S. Embassy

A PO 676

New York, N.Y.

Ames Rsch Center (NASA)

Technical Library

Moffett Field, Calif 94035 
High Speed Flight Center (NASA)

Technical Library

Edwards AFB, Calif 93523

Goddard Space Flight Center (NASA)

Greenbelt, Md. 20771

Geo. C. Marshall Space Flight Center (NASA)

Redstone Arsenal, Ala 35808

Lewis Research Center (NASA)

Technical Library

21000 Brookpark Road

Cleveland, Ohio

Aerospace Corp (Tech Library)

P.O. Box 95085

Los Angeles, Calif 90045

Rand Corporation

1700 Main St.

Santa Monica, Calif 90401

Carnegie Institute of Technology

Science \& Engineering Hunt Library

Schenley Park

Pittsburgh, $\mathrm{Pa} .15213$

California Institute of Technology

Aeronautics Library

1201 East Calif St.

Pasadena 4, Calif

AVCO Research Lab

Library

2385 Revere Beach Parkway

Everett, Mass 02149

Dr. G. E. Knausenberger

c/o Hq. Co. Munich Post

APO 09407

New York, N. Y.

Commander

Space Systems Division (AFSC)

Office of the Scientific Director

Inglewood, California

Commander

Aerospace Systems Division

AFSC

Office of the Scientific Director

Wright-Patterson AFB, Ohio

Commander

Aerospace Research Laboratories (OAR)

Office of the Scientific Director

Wright-Patterson AFB, Ohio
Commander

Air Force Cambridge Research Laboratories Office of the Scientific Director

L. G. Hanscom Field

Bedford, Massachusetts

Commander

Air Force Systems Command

Office of the Chief Scientist

Andrews AFB, Maryland

Commander

Research \& Technology Division AFSC

Office of the Scientific Director

Bolling AFB 25, D.C.

Commander

Rome Air Development Center

AFSC

Office of the Scientific Director

Griffiss AFB, Rome, New York

Department of the Navy

Dr. Arnold Shostak, Code 427

Head, Electronics Branch

Physical Sciences Division

Department of the Navy

Office of Naval Research

Washington, D.C. 20360

Chief of Naval Research, Code 427

Department of the Navy

Washington, D. C. 20360

Chief, Bureau of Weapons

Department of the Navy

Washington, D. C. 20360

Chief, Bureau of Ships

Department of the Navy

Washington, D. C. 20360

Attn: Code 680

Commander

U.S. Naval Air Development Center

Johnsville, Pennsylvania

Attn: NADC Library 
Library

U.S. Navy Electronics Laboratory

San Diego, California 92152

Commanding Officer

U.S. Navy Underwater Sound Laboratory

Ft Trumbull

New London, Connecticut

Director

Naval Research Laboratory

Washington, D.C. 20390

Commanding Officer

Office of Naval Research Branch Office

Navy 100, Fleet P.O. Box 39

New York, New York

Chief of Naval Operations

Pentagon OP 07T

Washington, D.C.

Commanding Officer

Officer of Naval Research Branch Office

495 Summer Street

Boston, Massachusetts 02110

Commander

Naval Ordnance Laboratory

White Oak, Maryland

Attn: Technical Library

U.S. Navy Post Graduate School

Monterey, California

Attn: Electrical Engineering Department 
